\title{
Description and Characterization of a Novel Method for Partial Volume Simulation in Software Breast Phantoms
}

\author{
Feiyu Chen, Predrag R. Bakic*, Member, IEEE, Andrew D. A. Maidment, Member, IEEE, \\ Shane T. Jensen, Xiquan Shi, and David D. Pokrajac
}

\begin{abstract}
A modification to our previous simulation of breast anatomy is proposed to improve the quality of simulated $x$-ray projections images. The image quality is affected by the voxel size of the simulation. Large voxels can cause notable spatial quantization artifacts; small voxels extend the generation time and increase the memory requirements. An improvement in image quality is achievable without reducing voxel size by the simulation of partial volume averaging in which voxels containing more than one simulated tissue type are allowed. The linear $x$-ray attenuation coefficient of voxels is, thus, the sum of the linear attenuation coefficients weighted by the voxel subvolume occupied by each tissue type. A local planar approximation of the boundary surface is employed. In the two-material case, the partial volume in each voxel is computed by decomposition into up to four simple geometric shapes. In the three-material case, by application of the Gauss-Ostrogradsky theorem, the 3D partial volume problem is converted into one of a few simpler 2D surface area problems. We illustrate the benefits of the proposed methodology on simulated x-ray projections. An efficient encoding scheme is proposed for the type and proportion of simulated tissues in each voxel. Monte Carlo simulation was used to evaluate the quantitative error of our approximation algorithms.
\end{abstract}

Manuscript received March 07, 2015; accepted April 01, 2015. Date of publication April 20, 2015; date of current version September 29, 2015. This project was supported by a grant from the US National Institutes of Health (R01 grant from the National Cancer Institute \#CA154444 and P20 grant from the National Institute of General Medical Sciences GM103446), the US Department of Defense Breast Cancer Research Program (HBCU Partnership Training Award \#BC083639), the US National Science Foundation (CREST grant \#HRD-0630388 and III grant \# 0916690), and the US Department of Defense/Department of Army (45395-MA-ISP, \#54412-CI-ISP, W911NF-11-2-0046). The content is solely the responsibility of the authors and does not necessarily represent the official views of the NIH, NSF and DoD. Asterisk indicates corresponding author.

F. Chen was with the Department of Mathematical Sciences, Delaware State University, Dover, DE 19901 USA. He is now with the College of Computer Science, Chongqing University, Chongqing 400044, China (e-mail: fchen@cqu. edu.cn).

*P. R. Bakic is with the Department of Radiology, University of Pennsylvania, Philadelphia, PA 19104 USA (e-mail: predrag.bakic@uphs.upenn.edu).

A. D. A. Maidment is with the Department of Radiology, University of Pennsylvania, Philadelphia, PA 19104 USA (e-mail: andrew.maidment@uphs.upenn.edu).

S. T. Jensen is with the Department of Statistics, The Wharton School, University of Pennsylvania, Philadelphia, PA 19104 USA (e-mail: stjensen@wharton.upenn.edu).

$X$. Shi is with the Department of Mathematical Sciences, Delaware State University, Dover, DE 19901 USA (e-mail: xshi@desu.edu).

D. D. Pokrajac is with the Department of Computer and Information Sciences, Delaware State University, Dover, DE 19901 USA (e-mail: dpokrajac@desu. edu).

Color versions of one or more of the figures in this paper are available online at http://ieeexplore.ieee.org.

Digital Object Identifier 10.1109/TMI.2015.2424854

Index Terms-Anthropomorphic breast phantom, digital mammography, Monte Carlo, partial volume simulation.

\section{NOMENCLATURE}

$A_{i}, i=1,2$

$\mathrm{b}$

$c^{\prime}$

$\mathrm{c}^{\prime \prime}$

$C_{i}, i=1, \ldots, \mathrm{K}$

d

$d_{i}, i=1,2$

$\mathrm{D}$

$f_{i}(\mathbf{x}), i=1, \ldots, \mathrm{K}$

$F_{i j}(\mathbf{x})$

$f_{M}(\mathbf{x})$

$f_{m}(\mathbf{x})$ distance of the simulated nipple point from the chest wall.

surface areas of the boundary of $V_{i}$ belonging to planes $\pi_{1}$ and $\boldsymbol{\pi}_{2}$

half of the uncompressed phantom thickness

vertical phantom dimension measured above the nipple level.

vertical phantom dimension measured below the nipple level.

simulated tissue compartments.

thickness of skin.

the distance between a vertex and planes.

thickness of the simulated Cooper's ligaments.

compartment shape functions.

difference of the compartment shape functions $\mathrm{f}_{i}(\mathbf{x})$ and $\mathrm{f}_{j}(\mathbf{x})$.

shape function defining the outer surface of the simulated skin layer.

shape function defining the inner surface of the simulated skin layer. 


\begin{tabular}{|c|c|c|}
\hline$M S E_{M C R}$ & $\begin{array}{l}\text { the mean square error } \\
\text { estimation of Monte Carlo } \\
\text { method using repetition. }\end{array}$ & $\mathrm{q}$ \\
\hline$M S E_{M C S}$ & $\begin{array}{l}\text { the mean square error } \\
\text { estimation of Monte Carlo } \\
\text { method based on sample } \\
\text { means. }\end{array}$ & $\mathrm{r}_{0}, \mathrm{r}_{s}$ \\
\hline$M S E_{q}$ & $\begin{array}{l}\text { the mean square quantization } \\
\text { error. }\end{array}$ & \\
\hline nPoint & $\begin{array}{l}\text { the number of intersections } \\
\text { between plane and edge(ext). }\end{array}$ & $\mathrm{S}_{i}, i=1,2,3$ \\
\hline $\begin{array}{l}n V \text { ertex }, n V \text { ertex } 1, \\
n V \text { ertex } 2\end{array}$ & $\begin{array}{l}\text { the number of vertices above } \\
\text { plane. }\end{array}$ & $t$ \\
\hline nVolume & $\begin{array}{l}\text { the number of geometrical } \\
\text { shapes need to be computed. }\end{array}$ & $\mathrm{T}$ \\
\hline $\mathbf{N}, \mathbf{N}_{1}, \mathbf{N}_{2}$ & $\begin{array}{l}\text { the normal vectors of } \\
\text { approximated planes. }\end{array}$ & $\begin{array}{l}\mathrm{V} \\
|V|\end{array}$ \\
\hline $\mathrm{N}_{\mathrm{MC}}$ & $\begin{array}{l}\text { the number of generated } \\
\text { random points of Monte } \\
\text { Carlo approach within a } \\
\text { voxel. }\end{array}$ & $\mathbf{x}_{0}, \mathbf{x}_{1}, \mathbf{x}_{2}$ \\
\hline $\mathrm{N}_{\mathrm{MCI}}$ & $\begin{array}{l}\text { the number of points that are } \\
\text { inside the measured volume. }\end{array}$ & $\mathbf{x}_{\mathrm{c}}$ \\
\hline $\mathrm{N}_{\text {repeat }}$ & $\begin{array}{l}\text { the number of repetitions } \\
\text { of the Monte Carlo method } \\
\text { applied on each voxel. }\end{array}$ & $\mathbf{x}_{\mathrm{m}}, \mathbf{x}_{\mathrm{M}}$ \\
\hline$p_{0}, p_{1}, p_{2}, p_{i}$ & $\begin{array}{l}\text { percentages of different } \\
\text { materials in the voxel. }\end{array}$ & $\Delta \mathrm{x}$ \\
\hline $\mathbf{P}_{i}, i=1, \ldots, 8$ & vertices of voxel V. & $\Delta \mathrm{x}^{\prime}$ \\
\hline $\begin{array}{l}\mathbf{P}, \mathbf{Q}, \mathbf{R}, \mathbf{S}, \mathbf{T}, \mathbf{U} \\
\mathbf{W}, \mathbf{X}, \mathbf{Y}, \mathbf{Z}\end{array}$ & $\begin{array}{l}\text { intersections between } \\
\text { approximation plane and } \\
\text { voxel edges. }\end{array}$ & $\varepsilon$ \\
\hline PV & $\begin{array}{l}\text { the sub-volume of voxel } \mathrm{V} \\
\text { above plane/planes. }\end{array}$ & \\
\hline $\mathrm{PV}_{j}, j=1, \ldots, T$ & $\begin{array}{l}\text { the true value of the partial } \\
\text { volume in voxel } j \text {. }\end{array}$ & $\varepsilon_{A}$ \\
\hline $\mathrm{PV}_{A, j}, j=1, \ldots, \mathrm{T}$ & $\begin{array}{l}\text { the linear approximation of } \\
\text { the partial volume in voxel } j \text {. }\end{array}$ & $\begin{array}{l}\varepsilon_{M} \\
\varepsilon_{M C}\end{array}$ \\
\hline $\mathrm{PV}_{M, j}, j=1, \ldots, T$ & $\begin{array}{l}\text { the approximation of the } \\
\text { partial volume in voxel } j \\
\text { using certain method } \mathrm{M} \text {. }\end{array}$ & $\varepsilon_{M C R, i j}$ \\
\hline $\mathrm{PV}_{M C, j}, j=1, \ldots, T$ & $\begin{array}{l}\text { the approximation of the } \\
\text { partial volume in voxel } j \\
\text { using Monte Carlo. }\end{array}$ & $\mu_{V}$ \\
\hline $\mathrm{PV}_{M C R, j}, j=1, \ldots, T$ & $\begin{array}{l}\text { the estimation of } \mathrm{PV}_{j} \text { using } \\
\text { Monte Carlo repetition in } \\
\text { voxel } j \text {. }\end{array}$ & $\begin{array}{l}\mu_{i} \\
\pi, \pi_{1}, \pi_{2}\end{array}$ \\
\hline $\begin{array}{l}\mathrm{PV}_{M C R, i j}, i= \\
1, \ldots \mathrm{N}_{\text {repeat }} ; j=1, \ldots, \mathrm{T}\end{array}$ & $\begin{array}{l}\text { the approximation of the } \\
i \text {-th repetition of the Monte } \\
\text { Carlo method applied on } j \text {-th }\end{array}$ & $\boldsymbol{\sigma}_{1}, \boldsymbol{\sigma}_{2}, \boldsymbol{\sigma}_{3}$ \\
\hline
\end{tabular}

$M S E_{M C R}$

$M S E_{M C S}$

$M S E_{q}$

nPoint

$n V$ ertex,$n V$ erte $x 1$,

$\mathbf{N}, \mathbf{N}_{1}, \mathbf{N}_{2}$

$\mathrm{N}_{\mathrm{MC}}$

$\mathbf{P}, \mathbf{Q}, \mathbf{R}, \mathbf{S}, \mathbf{T}, \mathbf{U}$, $\mathbf{W}, \mathbf{X}, \mathbf{Y}, \mathbf{Z}$

\section{PV} voxel. the number of bits to discretize percentage of a partial volume.

parameters related to compartment orientation and size.

subsampling factor for naïve reference method; also, a parameter of intersections.

surface areas of the boundary formed by the voxel sides.

the parameter of intersections.

the total number of partial volume voxels.

the symbol for a 3D voxel.

the volume of voxel $\mathrm{V}$.

the subvolume of material $i$ in the voxel $\mathrm{V}$.

the fixed points on approximating planes.

the center of voxel $\mathrm{V}$.

two vertices of the voxel, such that one of them is inside the skin and another one outside of skin, (used to compute $\mathbf{x}_{0}$ ).

linear dimension of voxel $\mathrm{V}$.

linear dimension of voxel $\mathrm{V}$ for naïve reference method.

difference between partial volumes computed by $\mathrm{M}$ and by linear approximation.

the error of linear approximation.

the error of method $\mathrm{M}$.

the error of Monte Carlo.

the estimate of the error of $i$-th repetition of the Monte Carlo approach on $j$-th voxel.

the X-ray attenuation in the voxel $\mathrm{V}$.

the X-ray attenuation of material $i$ in the voxel $\mathrm{V}$.

the linear approximations of boundaries between different materials.

planes corresponding to voxel sides. 


\section{INTRODUCTION}

$\mathbf{T}$ HIS study is motivated by a desire to improve the quality of synthetic images generated using software anthropomorphic breast phantoms. Software breast phantoms have received increasing attention for their use in preclinical validation of breast imaging systems and image analysis methods. Preclinical validation in the form of virtual clinical trials can improve the validation efficacy by identifying the most promising parameter settings to be assessed in a focused clinical trial. There are various designs of software breast phantoms, including phantoms developed using the rules for simulating anatomical structures [1]-[11] and phantoms based upon individual clinical 3D breast images [12]-[17].

The software anthropomorphic phantoms developed at the University of Pennsylvania have been used in various applications, including the validation and optimization of digital breast tomosynthesis (DBT) reconstruction methods [18]-[20], DBT image denoising methods [21], [22], ultrasound tomography (UST) reconstruction and segmentation methods [23], [24], analysis of power spectra descriptors in simulated phantom DBT images [10], [25], [26], analysis of texture properties in phantom digital mammography (DM) and DBT images [27], [28], and analysis of tumor detectability in DBT [29]-[31]. Physical versions of the $3 \mathrm{D}$ anthropomorphic software phantom have also been produced [32]-[36].

The current method for simulating breast anatomy [11] assumes that each voxel contains a single tissue type; this may cause notable artifacts due to abrupt attenuation transitions at the borders between regions of different simulated materials. The realism of the resulting phantom images is thus reduced. The realism can be improved by using a smaller voxel size. Reducing the voxel size, however, extends the phantom generation time and increases memory requirements. It should be possible to improve image quality without reducing voxel size by explicitly accounting for voxels containing more than one simulated tissue type.

Partial volume (PV) averaging can help reduce the quantization artifacts on boundaries of regions with different simulated materials. In PV averaging, voxels containing more than one simulated tissue type are allowed; thus, the linear x-ray attenuation coefficient of voxels is the sum of the linear attenuation coefficients weighted by the voxel subvolume occupied by each tissue type. The software phantoms in this study have been generated based upon the recursive partitioning of the phantom volume using octrees [11]. Previously, we reported about the development of a PV technique for selected tissue boundaries in our software breast phantoms [37], [38]. In our 2012 SPIE paper, PV simulation was introduced in phantom voxels containing up to two different simulated tissue types [37]. First, the PV of each voxel occupied by different materials was computed, and the linear attenuation coefficient values assigned as the linear combination of attenuations weighted by the PV occupied by each material in the voxel. These PVs could be also used to calculate the proportion of different materials accurately, both in individual voxels and the whole phantom. The same report discussed an encoding technique to accomplish efficient storage of the material composition. The initial results were illustrated using synthetic projections through phantoms with PV simulated on the skin-air boundary only.

In our 2012 IWDM paper [38], we proposed an extension to the PV simulation method to include voxels with three simulated materials. The PV was computed based upon a planar boundary approximation in voxels with multiple simulated tissue types. The improvement of image quality was qualitatively validated. The results were shown in the form of slices and simulated X-ray projections of phantoms with and without $\mathrm{PV}$, assuming a parallel beam of monoenergetic $\mathrm{X}$-rays without scatter.

Our current work is focused upon PV simulation of software phantoms generated based upon rules for simulating anatomical structures [7], [9], [11], [39]. PV simulation has been implicitly used to generate phantoms based upon computed tomography (CT) images of mastectomy specimen [13], [15], [17] or clinical breast CT data [14], [40]. These PV simulations arise naturally, because all raw volumetric images include partial volumes, as various tissues may contribute to the signal acquired in a single image voxel. In the simulation based upon mastectomy CT data [13], [15], [17], the values of each reconstructed breast CT image voxel were scaled into a value from 0.01 to 0.99 . The scaled values were interpreted as percentage of adipose tissue contained in the voxel. The scaling method resulted in phantom images more similar to the original CT data, as compared to the method based upon the segmentation into discrete tissue types. The scaling helped to preserve some of the fine tissue structure which would be lost when using the segmentation; however, it resulted in noisier images. The software phantoms developed using clinical CT data [14], [40] were designed by initially segmenting the $\mathrm{CT}$ data into voxels corresponding to skin, adipose tissue, and fibroglandular tissue. To improve realism, it was found to be necessary to segment the fibroglandular tissue into multiple classes based upon CT image intensity level; these classes were associated with different adipose-to-dense tissue volumetric ratios.

In this paper, we formulate the details of a PV simulation in the general case with up to three tissue types simulated in a voxel. A qualitative validation of the proposed method is performed in the slices through phantoms with PV simulated at different tissue interfaces. In addition, a direct validation is provided by the analysis of the difference between the PV estimates obtained with the proposed method vs. Monte Carlo estimates of PV. Finally, we present results from a qualitative analysis of phantom projections simulated using a polyenergetic divergent $\mathrm{x}$-ray beam approximation without scatter.

\section{Partial Volume Simulation Method}

\section{A. Breast Phantom Generation}

Breast phantoms in this work were generated utilizing the approach described in [11]. The simulated anatomy consists of compartments $\mathrm{C}_{i}, i=1, \ldots, \mathrm{K}$ and Cooper's ligaments $\mathrm{L}$, which separate the compartments from each other. The distribution, orientation and shapes of the compartments as well as the shape of the Cooper's ligaments are determined by pre-specified 
shape functions $f_{i}, i=1, \ldots, \mathrm{K}$. The proposed approach utilizes octrees to split the phantom volume $\mathrm{V}$ recursively. The recursive partitioning procedure begins with the root node, which is always flagged for splitting. For each level of the tree, we generate the nodes at the next level by recursively splitting the nodes flagged for splitting. The flagged nodes contain more than one material type. The recursive partitioning procedure continues until an individual node of the tree belongs to a single type (non-partial volume nodes) or until the maximal tree level is reached. The nodes corresponding to the maximal tree level correspond to the voxels. The breast outline and skin boundary are simulated with ellipsoidal surfaces, corresponding to the phantom volume vertically above and below the nipple level. The number of compartments $\mathrm{K}$, the shape functions, the skin thickness $\mathrm{d}$ and target thickness $\mathrm{D}$ of the Cooper's ligaments, and the voxel size $\Delta \mathrm{x}$ are input parameters of the algorithm.

\section{B. Different Cases of Phantom Voxels Containing Multiple Materials}

For realistic cases where $\Delta \mathrm{x}<\mathrm{D} / \sqrt{3}, \Delta \mathrm{x}<\mathrm{d} / \sqrt{3}$ the phantom voxels can be categorized as follows (see Fig. 1(a)):

A) Voxels, containing a single material: (1) skin; (2) air; (3) Cooper's ligament; (4) adipose tissue; and (5) fibroglandular dense tissue.

B) Partial volume voxels:

a) Voxels containing two materials (with one bounding surface): (6) skin and air; (7) skin and adipose tissue; (8) skin and dense tissue; (9) skin and Cooper's ligament; (10) Cooper's ligament and adipose tissue; (11) ligament and fibroglandular dense tissue;

b) Voxels containing three materials (with two bounding surfaces): (12) skin, ligament, and adipose tissue; and (13) skin, ligament, and dense tissue.

The effective linear $\mathrm{x}$-ray attenuation in a voxel which contains more than one simulated material, Fig. 1(b), can be calculated as:

$$
\begin{aligned}
\mu_{V} & =\frac{1}{|V|} \sum_{i} \mu_{i}\left|V_{i}\right|=\sum_{i} \mu_{i} p_{i} \\
p_{i} & =\frac{\left|V_{i}\right|}{|V|} \times 100 \%
\end{aligned}
$$

where $|V|$ is the voxel volume, $\left|V_{i}\right|$ is the subvolume of material $i$ with the linear x-ray attenuation $\mu_{i}$, and $p_{i}$ is the percentage of the material $i$ in the voxel.

The memory requirements for the phantom depend on ellipsoidal outline semiaxes and the voxel size $\Delta \mathrm{x}$. For efficient storage of the voxel material composition, we propose a representation of material types and percentages of the materials using a two-byte binary word. Since a voxel size smaller than the thickness of the skin or Cooper's ligaments is assumed, it is sufficient to consider combinations of up to three materials in a voxel. Thus, it suffices to store percentages of two materials $p_{1}$ and $p_{2}$. The percentage $p_{0}$ of the other material can be calculated by subtracting the stored percentages from $100 \%$, i.e.,

$$
p_{0}=100-p_{1}-p_{2}
$$

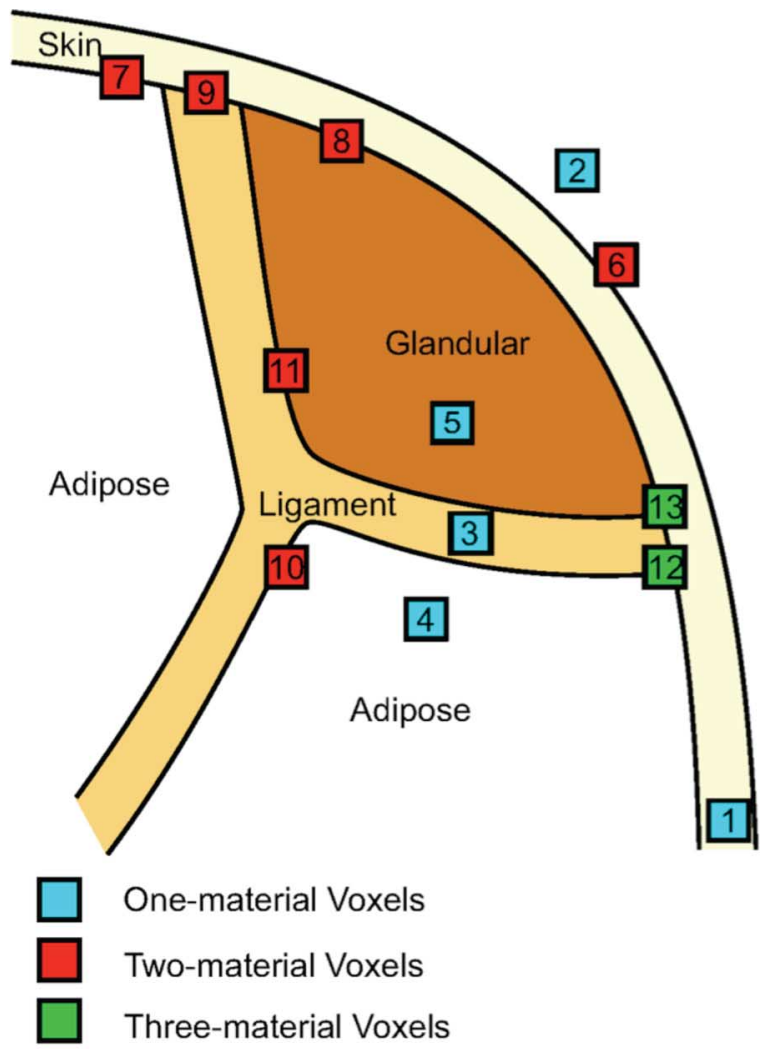

(a)

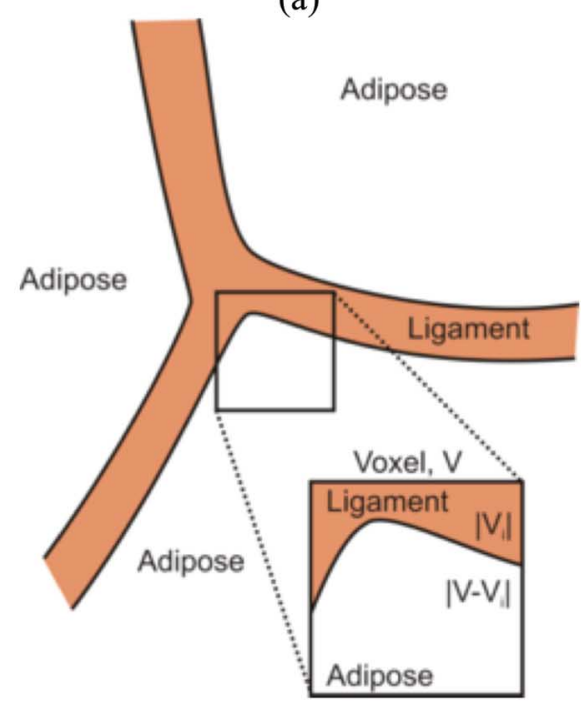

(b)

Fig. 1. (a) Taxonomy of material combinations in a voxel. (b) The concept of PV simulation; $V$ denotes the voxel volume and $V_{i}$ is the sub-volume occupied by dense tissue.

The interpretation of percentages $p_{0}, p_{1}$, and $p_{2}$ is specified by a four-bit voxel label, see Table I. The percentages $p_{1}$ and $p_{2}$ are stored as two records, $\mathrm{q}=6$ bits each. The choice of $\mathrm{q}$ is supported in our results (below); other values of q could be used with this schema as necessary. Using this representation schema, it is possible to encode all partial volume cases from the taxonomy discussed above, see Table II. For example, consider the case when the label $=0$ (skin/air boundary). Here, $p_{1}$ 
TABLE I

Encoding Partial Volume Material Percentages Using Four-Bit LABEL (Two BITS RESERVED For FutURE USE)

\begin{tabular}{cccc}
\hline \hline Label & $p_{0}$ & $p_{1}$ & $p_{2}$ \\
\hline 0 & skin & ligament & air \\
1 & ligament & fat & dense \\
2 & fat & ligament & skin \\
3 & dense & ligament & skin \\
\hline \hline
\end{tabular}

TABLE II

ENCODING TAXONOMY OF VOXELS

\begin{tabular}{llll}
\hline \hline \multicolumn{1}{c}{ Case } & $\begin{array}{c}p_{1}(6 \\
\text { bits })\end{array}$ & $\begin{array}{l}p_{2}(6 \\
\text { bits })\end{array}$ & $\begin{array}{c}\text { Label } \\
(4 \\
\text { bits })\end{array}$ \\
\hline 1. Skin & 0 & 0 & 0 \\
2. Air & 0 & $\begin{array}{l}p_{\text {Air }} \\
(=100)\end{array}$ & 0 \\
3. Cooper's ligament & 0 & 0 & 1 \\
4. Fat & 0 & 0 & 2 \\
5. Dense & 0 & 0 & 3 \\
6. Skin; air & 0 & $p_{\text {Air }}$ & 0 \\
7. Skin; fat tissue & 0 & $p_{\text {Skin }}$ & 2 \\
8. Skin; dense tissue & 0 & $p_{\text {Skin }}$ & 3 \\
9. Skin; Cooper's ligament & $p_{\text {Cooper }}$ & 0 & 0 \\
10. Cooper's ligament; fat & $p_{\text {Fat }}$ & 0 & 1 \\
11. Cooper's ligament; dense & 0 & $p_{\text {Dense }}$ & 1 \\
12. Skin, Cooper's ligament and fat tissue & $p_{\text {Cooper }}$ & $p_{\text {Skin }}$ & 2 \\
13. Skin, Cooper's ligament and dense & $p_{\text {Cooper }}$ & $p_{\text {Skin }}$ & 3 \\
tissue & & & 3 \\
\hline \hline
\end{tabular}

corresponds to Cooper's ligament tissue (with a constant value 0 in air/skin voxels), while $p_{2}$ corresponds to air (the ratio $\mid V$ $\left.-V_{i}|/| V \mid\right)$. The percentage $\mathrm{p}_{0}$ of skin, can be calculated from (2). The proposed representation also covers the cases when a voxel is comprised of a single material (e.g., a voxel belonging entirely to skin would have label 0 and $p_{1}=p_{2}=0$ ). The rest of this section discusses use of linear approximation to compute partial volumes $p_{i}$ in two- and three-material partial volume voxels.

\section{Partial Volume Computation for Two Material Voxels}

For voxels containing two materials, we compute a planar approximation of the boundary surface separating the materials. Subsequently, we calculate the portions of the voxel volume split by the planar approximation. Here we discuss the planar approximations for voxels containing skin (Cases 6-9) and voxels on ligament-compartment boundaries (Cases 10-11), followed by the computation of the voxel's volume above the plane.

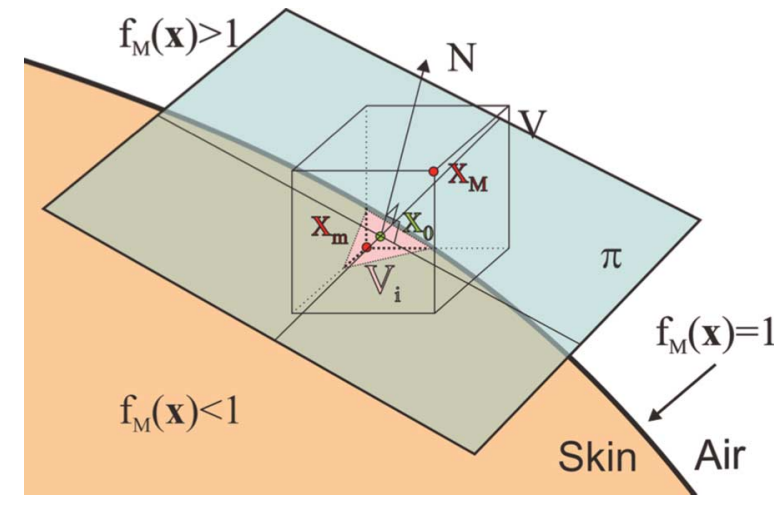

Fig. 2. Local approximation of skin boundary (defined by $\mathrm{f}_{M}(\mathbf{x})=1$ ) by a tangent plane.

1) Voxels Containing Skin (Cases 6-9; See Table II): We assume that the outer and the inner surface of the skin (skin/air and skin/tissue boundaries), are defined by functions $f_{M}(\mathbf{x})$ and $f_{m}(\mathbf{x})$ as follows [11]:

$$
\begin{gathered}
f_{m}(\mathrm{x})=f_{m}(x, y, z)=\left\{\begin{array}{l}
1, x<0 \\
\frac{x^{2}}{(a-d)^{2}}+\frac{y^{2}}{(b-d)^{2}}+\frac{z^{2}}{\left(c^{\prime}-d\right)^{2}}, x>0, z \geq 0, \\
\frac{x^{2}}{(a-d)^{2}}+\frac{y^{2}}{(b-d)^{2}}+\frac{z^{2}}{\left(c^{\prime \prime}-d\right)^{2}}, x>0, z<0
\end{array}\right. \\
f_{M}(\mathrm{x})=f_{M}(x, y, z)=\left\{\begin{array}{l}
1, x<0 \\
\frac{x^{2}}{a^{2}}+\frac{y^{2}}{b^{2}}+\frac{z^{2}}{c^{\prime 2}}, x>0, z \geq 0, \\
\frac{x^{2}}{a^{2}}+\frac{y^{2}}{b^{2}}+\frac{z^{2}}{c^{\prime \prime 2}}, x>0, z<0 .
\end{array}\right.
\end{gathered}
$$

Here we discuss in detail the computation of the partial volume for voxels containing skin and air (case 6). Other cases can be treated similarly (with function $f_{M}(\mathbf{x})$ appropriately replaced with $\left.f_{m}(\mathbf{x})\right)$. Since $f_{M}(\mathbf{x})$ is known in a closed form, the volume $\left|V_{\mathrm{i}}\right|$ can be exactly calculated; however, this calculation is computationally inefficient. Instead, the function $f_{M}(\mathbf{x})$ is approximated by a tangent plane $\boldsymbol{\pi}$ which reduces the considered problem to computation of the voxel volume below a pre-specified plane. The tangent plane $\pi$ on the surface $f_{M}(\mathbf{x})=1$ is placed at a point $\mathbf{x}_{0}$ inside the voxel $\mathrm{V}$. The point $\mathbf{x}_{0}$ satisfies $f_{M}(\mathbf{x})=1$ and is on the line segment between the points $\mathbf{x}_{m}$ and $\mathbf{x}_{M}$. The points $\mathbf{x}_{m}$ and $\mathbf{x}_{M}$ are calculated such that $\mathbf{x}_{m}=\arg \min _{V} f_{M}(\mathbf{x}), \mathbf{x}_{M}=\arg \max _{V} f_{M}(\mathbf{x})$. Points $\mathbf{x}$ on the tangent plane $\boldsymbol{\pi}$ satisfy: $\left(\mathbf{x}-\mathbf{x}_{0}\right) \cdot \mathbf{N}=0$, where denotes the scalar product and $\mathbf{N}=\nabla f_{M}\left(\mathbf{x}_{0}\right)$ is the gradient vector at the point $\mathbf{x}_{0}$ (See Fig. 2).

2) Voxels Containing Cooper's Ligaments and Compartmental Tissue (Cases 10-11; see Table II): The planar approximation for the boundary between the Cooper's ligaments and adipose tissue or dense tissue (cases 11 and 12) can be obtained as follows. Without loss of generality, consider adipose compartments $\mathrm{C}_{i}$ and $\mathrm{C}_{j}$ with corresponding shape functions $f_{i}$ and $f_{j}$. A Cooper's ligament between the compartments is the locus of points within a distance $\mathrm{D} / 2$ from a median surface $F_{i j}(\mathbf{x})=f_{i}(\mathbf{x})-f_{j}(\mathbf{x})=0$, see Fig. 3 . Consider a voxel V with center $\mathbf{x}_{\mathrm{c}}$. We define a planar approximation $\boldsymbol{\pi}_{\mathbf{1}}$ of the boundary between the Cooper's ligament and the compartment $\mathrm{C}_{j}$ as

$$
\boldsymbol{\pi}_{1}:\left(\mathbf{x}-\mathbf{x}_{1}\right) \cdot \mathbf{N}_{1}=0
$$




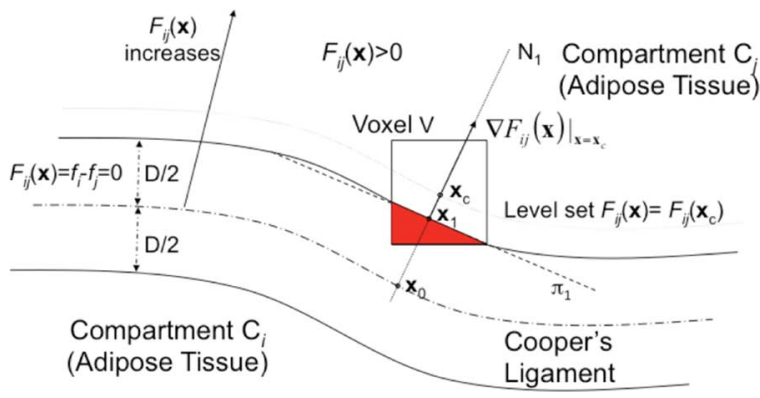

Fig. 3. Planar approximation of a boundary between Cooper's ligament and a compartment.

Here, $\mathbf{N}_{1}$ is a vector normal to a level set $F_{i j}(\mathbf{x})=F_{i j}\left(\mathbf{x}_{\mathbf{c}}\right)$ at $\mathbf{x}_{\mathbf{c}}$. $\mathbf{x}_{1}$ is a point on the normal at distance $\mathrm{D} / 2$ from the intersection of the normal and the median surface, such that:

$\mathbf{x}_{1}=\mathbf{x}_{\mathrm{c}}+\operatorname{sign}\left(F_{i j}\left(\mathbf{x}_{c}\right)\right)\left(\frac{D}{2}-\frac{F_{i j}\left(\mathbf{x}_{c}\right)}{\left\|\nabla F_{i j}\left(\mathbf{x}_{c}\right)\right\|}\right) \cdot \frac{\nabla F_{i j}\left(\mathbf{x}_{c}\right)}{\left\|\nabla F_{i j}\left(\mathbf{x}_{c}\right)\right\|}$,

$\mathbf{N}_{1}=\operatorname{sign}\left(F_{i j}\left(\mathbf{x}_{c}\right)\right) \nabla F_{i j}\left(\mathbf{x}_{c}\right)$.

3) Calculation of the Volume Above a Planar Approximation of two Materials Boundary: In this section, a fast and exact method is proposed to determine the fraction $\left|V_{i}\right| /|V|$ of the voxel's $\mathrm{V}$ volume located above the plane $\boldsymbol{\pi}$ (a case when $V_{i}$ is below the plane is reduced to this case by changing the direction of the normal vector of the plane). The first step of the method is to determine the number $n$ Vertex of voxel vertices above the plane. The voxel vertices $\mathbf{P}_{i}$ above the plane $\boldsymbol{\pi}$ specified by a normal $\mathbf{N}$ and containing a point $\mathbf{x}_{0}$ satisfy:

$$
\left(\mathbf{P}_{i}-\mathbf{x}_{0}\right) \cdot \mathbf{N}>0 .
$$

Depending upon $n$ Vertex, $\left|V_{i}\right|$ is computed using fundamental geometric shapes (e.g., prisms, prismoids or tetrahedrons, see Fig. 4). Note that if $n$ Vertex $>4$ computation of $\left|V_{i}\right|$ is reduced to computation of the complementary volume to the partial volume of $\mathrm{V}$ below the plane, see Algorithm A1 (Appendix A). The detailed algorithm for computation of partial volume of a voxel $\mathrm{V}$ above a given plane is specified in Algorithm A2.

The Algorithm A2 is very efficient. Observe that the considered partial volume problem reduces to 6 cases (Fig. 4). In each case, a small number of intersections (up to 9 ) between the plane $\boldsymbol{\pi}$ and voxel's edges (their extensions) need be calculated, see Table III, followed by computation of a volume of a geometric primitive.

Computation of intersections is also fast. To compute an intersection $\mathbf{Q}$ between an edge (extension of edge) containing vertices $\mathbf{P}_{i}$ and $\mathbf{P}_{j}$ and the plane $\boldsymbol{\pi}$, it is sufficient to resolve the system:

$$
\mathbf{Q}=\mathbf{P}_{i}+t\left(\mathbf{P}_{i}-\mathbf{P}_{j}\right)\left(\mathbf{Q}-\mathbf{x}_{0}\right) \cdot \mathbf{N}=0
$$

which results in the parameter $t$ specified by:

$$
t=-\frac{\left(\mathbf{P}_{i}-\mathbf{x}_{0}\right) \cdot \mathbf{N}}{\left(\mathbf{P}_{i}-\mathbf{P}_{j}\right) \cdot \mathbf{N}} .
$$

Since the vertices $\mathbf{P}_{i}$ and $\mathbf{P}_{j}$ differ in only one coordinate, this requires computation of only one scalar product

$$
\left(\left(\mathbf{P}_{i}-\mathbf{x}_{0}\right) \cdot \mathbf{N}\right) .
$$

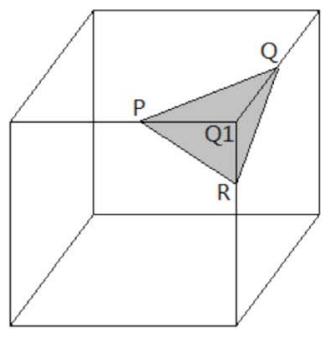

(a)

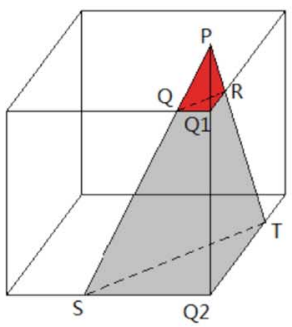

(c)

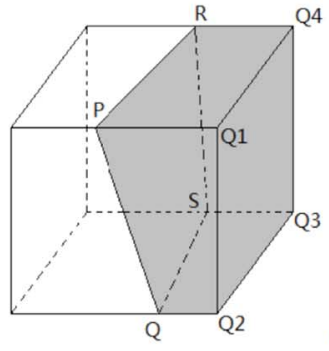

(e)

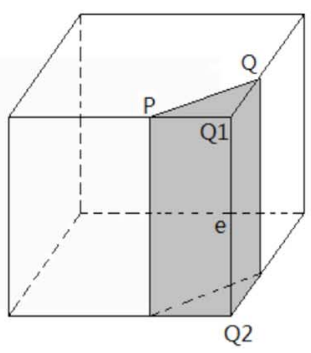

(b)

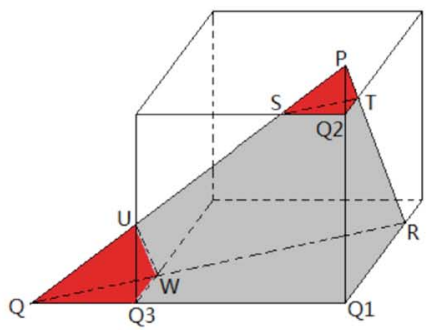

(d)

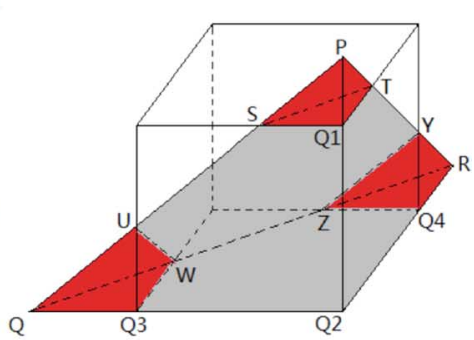

(f)
Fig. 4. Different cases of sub-volume.

TABLE III

Number of Vertices Above the Planar Approximation of the MATERIAL Boundary ( $\boldsymbol{n}$ Vertex), Number of INTERSECTIONS BETWEeN THE APproximation AND tHE VoXel EdGes or EdGE EXTENSIONS

\begin{tabular}{|c|c|c|c|c|c|c|}
\hline Case & A & B1 & B2 & C & D1 & D2 \\
\hline nVertex & 1 & \multicolumn{2}{|c|}{2} & 3 & \multicolumn{2}{|c|}{4} \\
\hline nPoint & 3 & 4 & 5 & 7 & 4 & 9 \\
\hline nVolume & $\begin{array}{c}1 \\
\text { tetra } \\
\text { hedron } \\
\end{array}$ & $\begin{array}{c}1 \\
\text { prism }\end{array}$ & $\begin{array}{c}2 \\
\text { tetra } \\
\text { hedron }\end{array}$ & $\begin{array}{c} \\
\text { tetra } \\
\text { hedron }\end{array}$ & $\begin{array}{c}1 \\
\text { prismoid }\end{array}$ & $\begin{array}{c}4 \\
\text { tetra } \\
\text { hedron }\end{array}$ \\
\hline
\end{tabular}
( $n$ Point) ANd Number of Volumes of Geometrical Primitives to Be COMPUTEd ( $n$ Volume), For DifFerent CASES of Algorithm A2 (SEE APPENDIX A).

The value of parameter $t$ depends on the position of $\mathbf{Q}$. If $\mathbf{Q}$ is located between $\mathbf{P}_{i}$ and $\mathbf{P}_{j}$, then $0<t<1$.

To compute intersection between $\boldsymbol{\pi}$ and other edges (extensions) we may proceed as follows. For an intersection $\mathbf{R}=$ $\mathbf{P}_{i}+s\left(\mathbf{P}_{i}-\mathbf{P}_{j}\right)$ between the plane and edge (extension of edge) containing $\mathbf{P}_{j}$ and $\mathbf{P}_{k}$, it is sufficient to compute

$$
s=-\frac{\left(\mathbf{P}_{k}-\mathbf{x}_{0}\right) \cdot \mathbf{N}}{\left(\mathbf{P}_{k}-\mathbf{P}_{j}\right) \cdot \mathbf{N}}=\frac{\left(\mathbf{P}_{i}-\mathbf{P}_{k}\right) \cdot \mathbf{N}-\left(\mathbf{P}_{i}-\mathbf{x}_{0}\right) \cdot \mathbf{N}}{\left(\mathbf{P}_{k}-\mathbf{P}_{j}\right) \cdot \mathbf{N}}
$$

which does not require computation of additional scalar products since $\mathbf{P}_{i}-\mathbf{P}_{j}$ only contains one non-zero coordinate.

The advantage of this procedure is that we can easily compute the partial volume without considering the shape of the boundary (interface) and the number of intersections between 


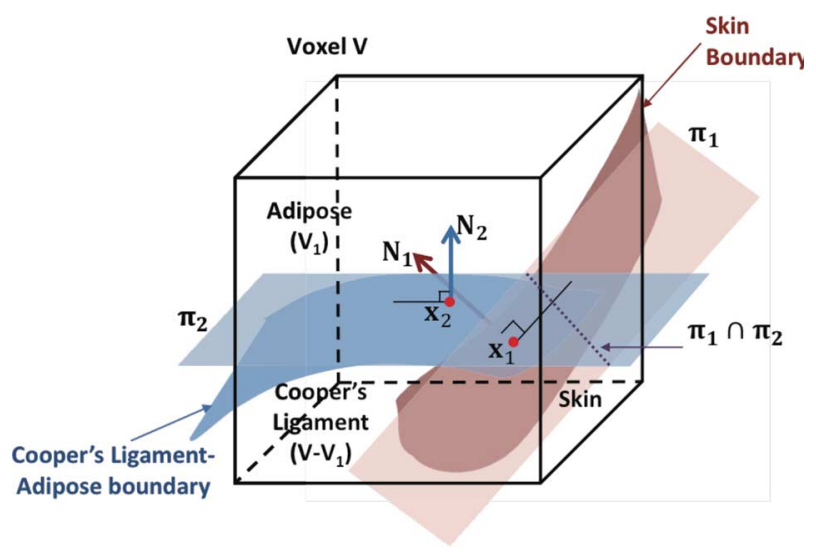

Fig. 5. An illustration of a three material voxel containing skin, Cooper's ligament and adipose tissue and planar approximations $\boldsymbol{\pi}_{1}$ and $\boldsymbol{\pi}_{2}$ of the tissue boundaries.

the plane and voxel. The cases are distinguished based on $n$ Vertex, that can be obtained easily.

4) Partial Volume Computation for Three Material Voxels (Cases 12, 13; See Table II): For a voxel V containing three materials we construct a planar approximation for each bounding surface (Fig. 5). The results of the approximation are planes $\boldsymbol{\pi}_{1:}\left(\mathbf{x}-\mathbf{x}_{1}\right) \cdot \mathbf{N}_{1}=0$ and $\boldsymbol{\pi}_{2}:\left(\mathbf{x}-\mathbf{x}_{2}\right) \cdot \mathbf{N}_{2}=0$. Here, $\boldsymbol{\pi}_{1}$ and $\boldsymbol{\pi}_{2}$ are linear approximations of the inner skin boundary and the ligament's boundary, respectively. The partial volume $\left|V_{i}\right|$ of interest is subsequently calculated as the volume of a portion of the voxel $\mathrm{V}$ that is below/above the planes. For example, the partial volume $V_{i}$ corresponding to the adipose tissue in Fig. 5 is computed as a volume of a part of the voxel that is both above planes $\pi_{1}$ and $\boldsymbol{\pi}_{2}$.

Given the planar approximations $\boldsymbol{\pi}_{1}$ and $\boldsymbol{\pi}_{2}$ of the material boundaries, we compute the partial volume $\left|V_{i}\right|$ using the divergence (i.e., Gauss-Ostrogradsky) theorem [41], [42]. Without loss of generality, we consider the volume $\mathrm{V}_{i}$ that is above both planes $\boldsymbol{\pi}_{1}$ and $\boldsymbol{\pi}_{2}$ (other cases can be treated by changing directions of vectors specifying $\boldsymbol{\pi}_{1}$ and $\boldsymbol{\pi}_{2}$ ). The divergence theorem can be stated as the following integral equation:

$$
\iint_{V_{i}} \int(\nabla \cdot F) d V=\oiint_{S}(F \cdot \mathbf{N}) d S .
$$

The left side is a volume integral of a vector field $F(\mathbf{x})$ over the partial volume $V_{i}$, the right side is the surface integral over the boundary of the volume $V_{i}$, and $\mathbf{N}$ is the outward pointing unit normal vector of the boundary. Note that the volume $V_{i}$ is bounded by planes $\boldsymbol{\pi}_{1}$ and $\boldsymbol{\pi}_{2}$ and at most 6 sides of the voxel.

The application of the divergence theorem depends on whether there is a voxel vertex $\mathbf{Q}_{1}$ above both planes $\boldsymbol{\pi}_{1}$ and $\boldsymbol{\pi}_{2}$. Assume that such a vertex $\mathbf{Q}_{1}$ exists. By choosing $F(\mathbf{x})=\mathbf{x},(9)$ reduces to:

$$
3\left|V_{i}\right|=\left(S_{1}+S_{2}+S_{3}\right) \Delta x+A_{\pi 1} d_{1}+A_{\pi 2} d_{2}
$$

where $S_{i}, i=1, \ldots, 3$ are surface areas of the boundary formed by the voxel sides $\sigma_{1}, \sigma_{2}$ and $\sigma_{3}$ that do not contain the vertex

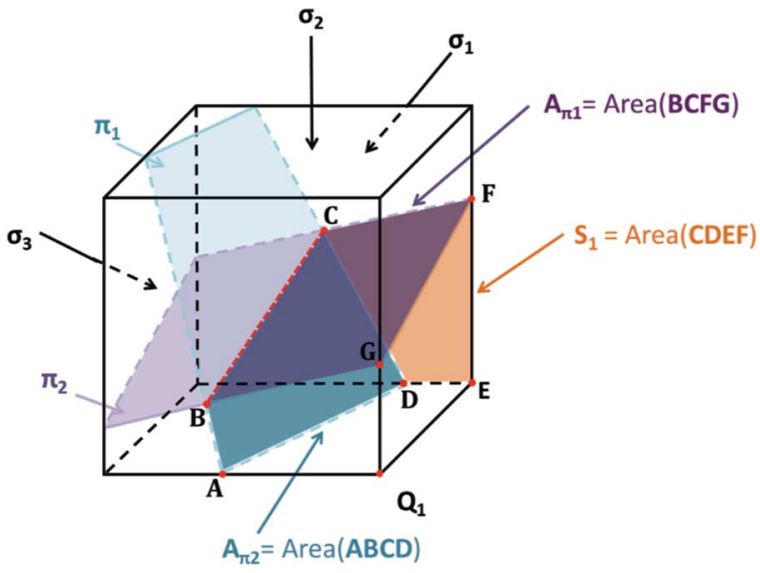

Fig. 6. Partial volume $V_{i}$ of the voxel $V$ above planes $\boldsymbol{\pi}_{1}$ and $\boldsymbol{\pi}_{2}$ and containing vertex $\mathbf{Q}_{1} . \mathrm{S}_{1}, \mathrm{~S}_{2}$ and $\mathrm{S}_{3}$ (here $\mathrm{S}_{2}=\mathrm{S}_{3}=0$ ) are surface areas of parts of the volume boundary belonging to voxel sides $\boldsymbol{\sigma}_{1}, \boldsymbol{\sigma}_{2}$ and $\boldsymbol{\sigma}_{3}$ that do not contain the vertex $\mathbf{Q}_{1}$.

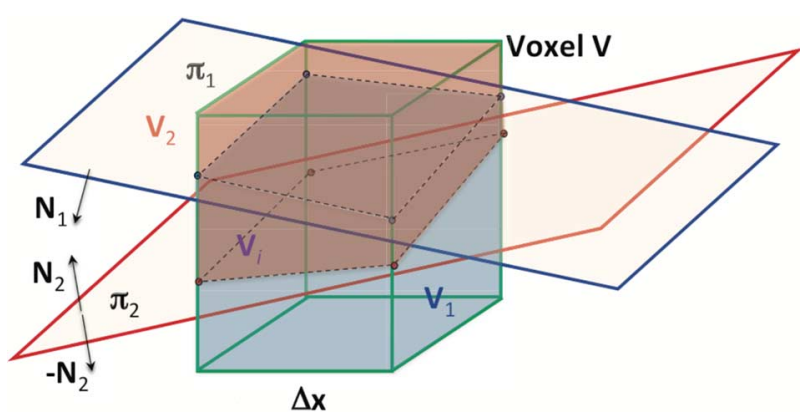

Fig. 7. Illustration of a case when there is no vertex of a voxel $\mathrm{V}$ above both planes $\boldsymbol{\pi}_{1}$ and $\boldsymbol{\pi}_{2}$ but the partial volume is larger than zero. The volume $V_{i}$ is the intersection of $V_{1}$ and $V_{2}$.

$\mathbf{Q}_{1} ; A_{\pi 1}$ and $A_{\pi 2}$ are surface areas of the boundary of $V_{i}$ belonging to planes $\boldsymbol{\pi}_{1}$ and $\boldsymbol{\pi}_{2}$ and $d_{1}=\left(\mathbf{Q}_{1}-\mathbf{x}_{1}\right) \cdot \mathbf{N}_{1}$, and $d_{2}=\left(\mathbf{Q}_{1}-\mathbf{x}_{2}\right) \cdot \mathbf{N}_{2}$ are distances of the vertex $\mathbf{Q}_{\mathbf{1}}$ to planes $\pi_{1}$ and $\boldsymbol{\pi}_{2}$ (see Fig. 6). Subsequently, the PV is calculated as:

$$
\left|V_{i}\right|=\frac{\left(S_{1}+S_{2}+S_{3}\right) \Delta x+A_{\pi 1} d_{1}+A_{\pi 2} d_{2}}{3} .
$$

If there is no vertex of $\mathrm{V}$ above both planes $\boldsymbol{\pi}_{1}$ and $\boldsymbol{\pi}_{2}$, it is still possible that $\left|V_{i}\right|>0$. As illustrated in Fig. 7, this is the case when the sets of vertices above $\boldsymbol{\pi}_{1}$ and $\boldsymbol{\pi}_{2}$ are both non empty. In such a case, partial volume can be computed as the difference of partial volumes above one of the planes (e.g., plane $\boldsymbol{\pi}_{1}$ ), calculated using Algorithm A1) the partial volume above $\boldsymbol{\pi}_{1}$ and below $\boldsymbol{\pi}_{2}$ (calculated by changing the direction of the normal vector $\mathbf{N}_{2}$ ). Note that in Fig. 7, the same approach is applicable in an adjacent voxel (here, right to $V$ ) where the planes $\boldsymbol{\pi}_{1}$ and $\boldsymbol{\pi}_{2}$ cross.

The Algorithm A3 (Appendix A) for computation of voxel partial volume above two planes is also very efficient. The $3 \mathrm{D}$ partial volume problem is converted into the computation of the linear combinations of a few 2D polygon areas. The polygon's vertices are chosen from the vertices of the voxel; the intersections of an edge of voxel and the plane; or the intersections of two planes on a voxel side $\boldsymbol{\sigma}_{i}, i=1,2,3$. The intersection of an edge of the voxel and the plane can be solved using (8). 


\section{VALidATION TECHNiQUES}

We have performed two validations of the proposed technique. First, we tested to what extent the proposed technique of linear approximation was capable of adequately representing the true values of partial volumes. This was performed by quantitative validation of the algorithm. Second, we evaluated the improvement of image quality by visual assessment of simulated images of phantoms with PV.

\section{A. Accuracy Assessment of the PV Computation}

The goal of qualitative validation of the proposed algorithm for PV approximation is to estimate the expectation of the squared error, i.e., $\mathrm{E}\left(\varepsilon_{A}^{2}\right)$, where, for each partial volume voxel $j$, the estimation error is defined as:

$$
\varepsilon_{\mathrm{A}, j}=\mathrm{PV}_{j}-\mathrm{PV}_{\mathrm{A}, j} .
$$

Here $\mathrm{PV}_{\mathrm{A}, j}$ is the partial volume in voxel $j$ obtained using the proposed method (a linear approximation) and $\mathrm{PV}_{j}$ is the true value of the partial volume, where (see Section III) both $\mathrm{PV}_{j}$ and $\mathrm{PV}_{\mathrm{A}, j}$ belong to a range $[0,1]$. However, a practical problem is that the true partial volumes $\mathrm{PV}_{j}$ are not directly observable, hence $\varepsilon_{\mathrm{A}, j}$ cannot be directly computed.

Consider a reference method $\mathrm{M}$ that can estimate the partial volume $\mathrm{PV}_{M, j}$ for each voxel $j$. At voxel $j$, we can only directly observe the difference $\varepsilon_{j}$ between partial volumes computed by method $\mathrm{M}$ and by the linear approximation:

$$
\varepsilon_{j}=\mathrm{PV}_{\mathrm{M}, j}-\mathrm{PV}_{\mathrm{A}, j} .
$$

We can easily obtain that:

$$
\varepsilon_{j}=\varepsilon_{\mathrm{M}, j}+\varepsilon_{\mathrm{A}, j}
$$

where:

$$
\varepsilon_{\mathrm{M}, j}=\mathrm{PV}_{\mathrm{M}, j}-\mathrm{PV}_{j}
$$

From (13):

$$
\mathrm{E}\left(\varepsilon_{\mathrm{A}}^{2}\right)=\mathrm{E}\left(\varepsilon^{2}\right)-\mathrm{E}\left(\varepsilon_{\mathrm{M}}^{2}\right)-2 \mathrm{E}\left(\varepsilon_{\mathrm{M}} \varepsilon_{\mathrm{A}}\right)
$$

Note that the definition of $\mathrm{E}\left(\varepsilon_{\mathrm{A}}{ }^{2}\right)$ guarantees that both sides of (15) are always non-negative. Also, note that the expectations are calculated over one particular phantom. In order to use a reference method $\mathrm{M}$ to estimate $\mathrm{E}\left(\varepsilon_{\mathrm{A}}{ }^{2}\right)$ using (13), one should be able to estimate the squared error of the reference method, $\mathrm{E}\left(\varepsilon_{\mathrm{M}}{ }^{2}\right)$. Also, the errors of the proposed approximation and the reference method should be uncorrelated, i.e., $\mathrm{E}\left(\varepsilon_{\mathrm{M}} \cdot \varepsilon_{\mathrm{A}}\right)=0$.

A naïve choice for the reference method $M$ is estimation of the PV based on subsampling. Let $\mathrm{P}$ be a considered partial volume phantom (with linear voxel dimension $\Delta \mathrm{x}$ ). Consider a non-partial volume phantom $\mathrm{P}^{\prime}$ that simulates an identical anatomy as $\mathrm{P}$, with linear voxel dimension $\Delta \mathrm{x}^{\prime}=\Delta \mathrm{x} / \mathrm{s}$ where $\mathrm{s}$ is an integer subsampling factor. For each voxel $j$, the partial volume can be estimated as the fraction of the corresponding voxels from $\mathrm{P}^{\prime}$ that contain the material of interest. Unfortunately, the subsampling method is not suitable as a reference method. First, in this method, $\mathrm{E}\left(\varepsilon_{\mathrm{M}}{ }^{2}\right)$ cannot be easily estimated. Second, errors of the proposed approximation and the reference method are correlated (e.g., $\varepsilon_{\mathrm{M}}$ is larger when the boundaries between different materials are more non-linear, i.e., where $\varepsilon_{A}$ is larger). Finally, the method may not be feasible, since the computation of a phantom $\mathrm{P}^{\prime}$ for large $s$ may be computationally prohibitive.

To overcome these difficulties, we propose to utilize a Monte Carlo approach [43] which gives us the opportunity to compare the precision of our PV approximation with a reference method based on Monte Carlo simulation. For each partial volume voxel $j$, we estimate $\mathrm{PV}_{\mathrm{MC}, \mathrm{j}}$ as follows. We generate $\mathrm{N}_{\mathrm{MC}}$ random points $\mathbf{x}$ within a voxel and determine the number $\mathrm{N}_{\mathrm{MCI}}$ of points that are inside the measured partial volume. Note that for voxels from cases 6-9 (see Section III-C1) this includes computing functions $f_{M}(\mathbf{x})\left(f_{m}(\mathbf{x})\right)((3),(4))$. For PV voxels containing ligament tissue (cases 10-13) we, in addition, need to determine the exact distance between $\mathbf{x}$ and the median surface (see Section III-C2); this can be done, e.g., using the algorithm described in [44]. The partial volume is subsequently obtained as

$$
\mathrm{PV}_{\mathrm{MC}, j}=\frac{\mathrm{N}_{\mathrm{MCI}}}{\mathrm{N}_{\mathrm{MC}}} .
$$

The error of the Monte Carlo method is defined as:

$$
\varepsilon_{\mathrm{MC}, j}=\mathrm{PV}_{\mathrm{MC}, j}-\mathrm{PV}_{j} .
$$

As shown in the Appendix B, (A8), $\mathrm{E}\left(\varepsilon_{\mathrm{MC}} \varepsilon_{\mathrm{A}}\right)=0$.

Therefore, from (15) we obtain:

$$
\mathrm{E}\left(\varepsilon_{\mathrm{A}}^{2}\right)=\mathrm{E}\left(\varepsilon^{2}\right)-\mathrm{E}\left(\varepsilon_{\mathrm{MC}}^{2}\right)
$$

$\mathrm{E}\left(\varepsilon^{2}\right)$ can be estimated using the sample mean square error (MSE):

$$
\mathrm{E}\left(\varepsilon^{2}\right) \sim M S E_{\text {total }}=\frac{1}{\mathrm{~T}} \Sigma_{j=1}^{\mathrm{T}} \varepsilon_{j}^{2}
$$

where $\mathrm{T}$ denotes the total number of partial volume voxels. The following subsection discusses estimates $M S E_{M C}$ of $\mathrm{E}\left(\varepsilon_{\mathrm{MC}}{ }^{2}\right)$. According to (18), (19) we estimate $\mathrm{E}\left(\varepsilon_{A}{ }^{2}\right)$ as:

$$
M S E_{A}=M S E_{\text {total }}-M S E_{M C} .
$$

Note that the computed partial volumes are quantized using $\mathrm{q}$ bits. Hence, $\mathrm{E}\left(\varepsilon_{A}{ }^{2}\right)$ is bounded by the quantization error. Under the assumption that $\mathrm{PV}_{M, j}$ are uniformly distributed within each quantization interval [45], the quantization error $M S E_{q}$ is approximated as:

$$
M S E_{q}=\frac{1}{12\left(2^{\mathrm{q}}-1\right)^{2}} .
$$

1) Estimation of MSE of Monte Carlo Approach: To ensure reliability of the validation, we utilize two techniques to estimate MSE of Monte Carlo approach. The first technique repeats the Monte Carlo process for each voxel in order to assess the true value of $\varepsilon_{\mathrm{MC}}$. The second technique is based on estimation of sample means of computed $\mathrm{PV}_{\mathrm{MC}}$ and completely avoids estimation of $\varepsilon_{\mathrm{MC}}$.

MSE of Monte Carlo Based on Estimating $\varepsilon_{M C}$ : Consider voxels belonging to one specific case of partial volume (e.g., two-material voxels on the ligament-compartment boundary). For each partial volume voxel of a particular phantom, we repeat the estimation of PV using the Monte Carlo approach $\mathrm{N}_{\text {repeat }}$ times. Denote the obtained estimates in the $i$-th repetition of the 
Monte Carlo method applied on $j$-th voxel as $\mathrm{PV}_{M C R, i j}(i=$ $\left.1, \ldots, \mathrm{N}_{\text {repeat }}, j=1, \ldots, \mathrm{T}\right)$. The idea of this approach is to obtain the estimate of the true partial volume by averaging these estimates.

Since the MC estimation is unbiased (see (A1)), we can use the following estimate of the true partial volume $\mathrm{PV}_{j}$ for the $j$-th voxel:

$$
\mathrm{PV}_{M C R, j}=\frac{1}{\mathrm{~N}_{\text {repeat }}} \sum_{i=1}^{\mathrm{N}_{\text {repeat }}} \mathrm{PV}_{M C R, i j}
$$

Based on this, the estimate $\varepsilon_{M C R, i j}$ of the error of the $i$-th repetition of the Monte Carlo approach on $j$-th voxel is:

$$
\varepsilon_{M C R, i j}=\mathrm{PV}_{M C R, i j}-\mathrm{PV}_{M C R, j} .
$$

Therefore, the MSE of MC can be estimated as:

$$
\begin{aligned}
& M S E_{M C R}=\frac{1}{\mathrm{TN}_{\text {Repeat }}} \sum_{j=1}^{\mathrm{T}} \sum_{i=1}^{\mathrm{N}_{\text {Repeat }}} \varepsilon_{M C R, i j}^{2} \\
& =\frac{1}{\mathrm{TN}_{\text {repeat }}} \sum_{j=1}^{\mathrm{T}} \sum_{i=1}^{\mathrm{N}_{\text {repeat }}}\left(\mathrm{PV}_{M C R, i j}-\mathrm{PV}_{M C R, j}\right)^{2} .
\end{aligned}
$$

In practice, it may be more computationally efficient to utilize the following formula:

$$
\begin{aligned}
M S E_{M C R}=\frac{1}{\mathrm{TN}_{\text {repeat }}} & \sum_{j=1}^{\mathrm{T}}\left[\sum_{i=1}^{\mathrm{N}_{\text {Repeat }}} \mathrm{PV}_{M C R, i j}^{2}\right. \\
& \left.-\frac{1}{\mathrm{~N}_{\text {repeat }}}\left(\sum_{i=1}^{\mathrm{N}_{\text {Repeat }}} \mathrm{PV}_{M C R, i j}\right)^{2}\right] .
\end{aligned}
$$

Estimation Based on Sample Means of $P V_{M C}$ : Here we demonstrate that an estimate of $\mathrm{E}\left(\varepsilon_{\mathrm{MC}}{ }^{2}\right)$ can be obtained by estimating $\mathrm{E}\left(\mathrm{PV}_{M C}\right)$ and $\mathrm{E}\left(\mathrm{PV}_{M C}{ }^{2}\right)$ (using a single Monte Carlo run $\left(\mathrm{N}_{\text {repeat }}=1\right)$ ). Note that this approach does not require knowledge of true values of $\mathrm{PV}_{j}$ at each voxel nor the availability of distribution $p(\mathrm{PV})$.

From (17) follows:

$$
E\left(\mathrm{PV}^{2}\right)=E\left(\mathrm{PV}_{M C}^{2}\right)-E\left(\varepsilon_{M C}^{2}\right)-2 E\left(\varepsilon_{M C} \mathrm{PV}\right)
$$

which due to (A5) in the Appendix B reduces to:

$$
E\left(\mathrm{PV}^{2}\right)=E\left(\mathrm{PV}_{M C}^{2}\right)-E\left(\varepsilon_{M C}^{2}\right) \text {. }
$$

By substituting $E(\mathrm{PV})$ and $E\left(\mathrm{PV}^{2}\right),(\mathrm{A} 7)$ and (27), into rightmost part of (A4) and solving for $E\left(\varepsilon_{M C}^{2}\right)$, we obtain:

$$
E\left(\varepsilon_{M C}^{2}\right)=\frac{1}{\mathrm{~N}_{\mathrm{MC}}-1}\left(E\left(\mathrm{PV}_{M C}\right)-E\left(\mathrm{PV}_{M C}^{2}\right)\right) \text {. }
$$

We estimate $E\left(\varepsilon_{M C}^{2}\right)$ using $M S E_{M C S}$ computed by utilizing sample means of Monte Carlo estimations of the partial volume and of the squared partial volume as:

$$
\begin{aligned}
M S E_{M C S}= & \frac{1}{\mathrm{~N}_{\mathrm{MC}}-1} \\
& \times\left(\frac{1}{\mathrm{~T}} \Sigma_{j=1}^{\mathrm{T}} P V_{M V_{j}}-\frac{1}{\mathrm{~T}} \Sigma_{j=1}^{\mathrm{T}} P V_{M V_{j}}{ }^{2}\right) .
\end{aligned}
$$

Note that $M S E_{M C S}$ depends on the estimated moments of $\mathrm{PV}_{M C}$, and hence may indirectly depend on the partial volume case (see Section III-B).

\section{B. Image Quality Improvement After PV Simulation}

All the simulations were implemented using Matlab (64-bit, MathWorks, Natick, MA). Phantoms were simulated on a computer with two Intel Xeon 5650 Six Core Processors (Intel, Inc., Santa Clara, CA) working at $2.53 \mathrm{GHz}$ with $128 \mathrm{~GB}$ RAM (1333 $\mathrm{MHz}$ DDR III ECC ) and utilizing one core per phantom. We used Matlab version v7.13(R2011b).

We generated $450 \mathrm{ml}$ phantoms (approximately a B cup bra size), [46] with ellipsoidal outline semiaxes $\mathrm{a}=\mathrm{b}=\mathrm{c}^{\prime \prime}=$ $5 \mathrm{~cm}, \mathrm{c}^{\prime}=12 \mathrm{~cm}$, (see [11], (2) and (3)). The voxel sizes were $100 \mu \mathrm{m}$ and $200 \mu \mathrm{m}$. The number of compartments was $\mathrm{K}=333$ [11]. The memory requirements are: 850MB $(100 \mu \mathrm{m}$ non-PV phantom), 212.5 MB (200 $\mu \mathrm{m} \mathrm{PV),} \mathrm{and} \mathrm{106.25} \mathrm{MB}$ (200 $\mu \mathrm{m}$ non-PV).

We specified the skin thickness $\mathrm{d}=1.5 \mathrm{~mm}$ based upon reports in the literature and the target thickness of the Cooper's ligaments $\mathrm{D}=0.6 \mathrm{~mm}$ [47], [48]. There are no explicit quantitative reports in the literature on the measured thickness of Cooper's ligaments in clinical data. We assumed the thickness was smaller than $1 \mathrm{~mm}$, as observed from subgross breast histological sections (e.g., the sections shown in[39]). Also, we varied the relative random compartment orientation $r_{0}$ and the relative compartment size, $\mathrm{r}_{s}$ [49].

Mammographic images of the phantom are simulated using (i) a finite-element model of mammographic breast compression, and (ii) simulation of the x-ray projections through the compressed phantom. The deformation model is implemented using Abaqus (version 6.6, DS Simulia Corp., Providence, RI), and is based upon a finite element model of breast compression proposed by Ruiter et al. [50]. The deformation model assumes the volume of the simulated breast tissue is preserved. With that assumption, a $450 \mathrm{ml}$ phantom described in Section III-A corresponds to a compressed phantom with a size of $20 \mathrm{~cm}$ in the vertical direction, $5 \mathrm{~cm}$ in the lateral direction, and approximately $6.5 \mathrm{~cm}$ in the chest wall-nipple direction.

Mammographic projections of the compressed phantom are simulated assuming a polyenergetic x-ray acquisition model without scatter. The quantum noise was simulated by a random Poison process, corresponding to the standard radiation dose of a clinical mammographic projection. The linear x-ray attenuation coefficients of the simulated tissues were selected using their energy dependence as listed in the NIST X-ray Mass Attenuation Tables [51]. The simulated acquisition geometry uses a source-detector distance of $70 \mathrm{~cm}$, a detector element size of $70 \mu \mathrm{m}$, and a $24 \mathrm{~cm} \times 30 \mathrm{~cm}$ field-of-view, corresponding to the Hologic Selenia Dimensions full-field digital mammography system (Hologic, Bedford, MA).

\section{Results}

\section{A. Qualitative Evaluation}

Fig. 8 illustrates the PV simulation in a $450 \mathrm{ml}$ software breast phantom with $200 \mu \mathrm{m}$ voxels, relative compartment orientation, $r_{0} \in[0.5,2]$ and relative compartment size, $\mathrm{r}_{s} \in[0.5,2]$. 

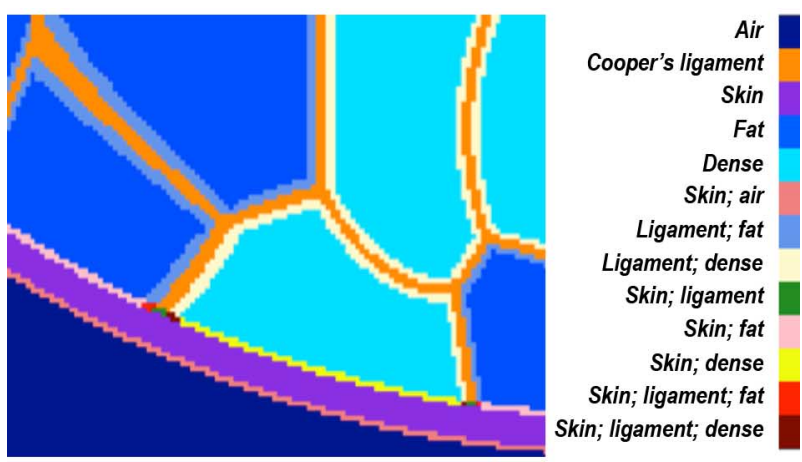

(a)

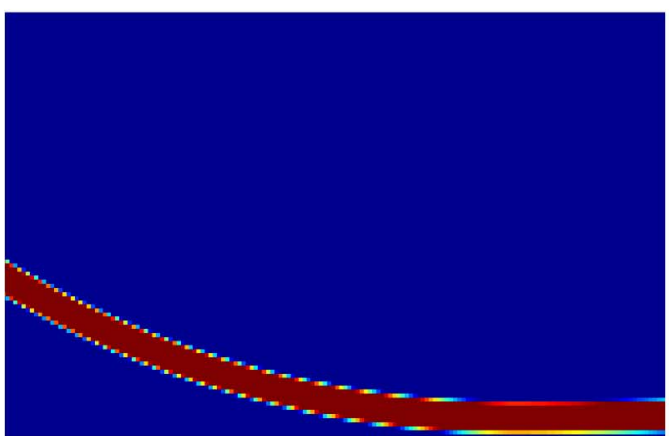

(b)

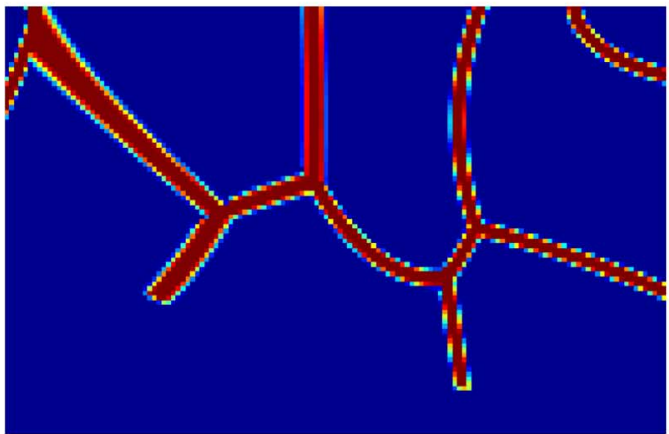

(c)

Fig. 8. (a) Various cases of PV voxels simulated by the proposed method in a slice of a $450 \mathrm{ml}$ software breast phantom, with $200 \mu \mathrm{m}$ voxels. Color-coded percentage of the skin (b) or Cooper's ligaments (c) have been computed in phantom voxels from (a).

Shown is the segmentation of phantom detail into air and voxels containing one, two or three materials.

Fig. 9 shows simulated x-ray projections of phantoms with and without simulated PV. The simulated acquisition assumed a polyenergetic x-ray beam and divergent x-ray beam. The projections correspond to three phantoms with identical distributions of compartments: a phantom with $200 \mu \mathrm{m}$ voxels and no PV (Fig. 9(a)); a $200 \mu \mathrm{m}$ phantom with simulated PV (Fig. 9(b)); and a phantom with $100 \mu \mathrm{m}$ voxels and no PV (Fig. 9(c)). Fig. 9(d) contains magnified detail of Fig. 9(a). Corresponding magnified details of Figs. 9(b) and 9(c) are shown in Figs. 9(e) and 9(f), respectively. Fig. 10 illustrates the effect of simulating PV with respect to the skin, two-material voxels, and three-material voxels, as seen in differences of projections.

\section{B. Quantitative Validation}

For quantitative validation of the proposed method, we utilized the Monte Carlo method, as described in Section IV-A.

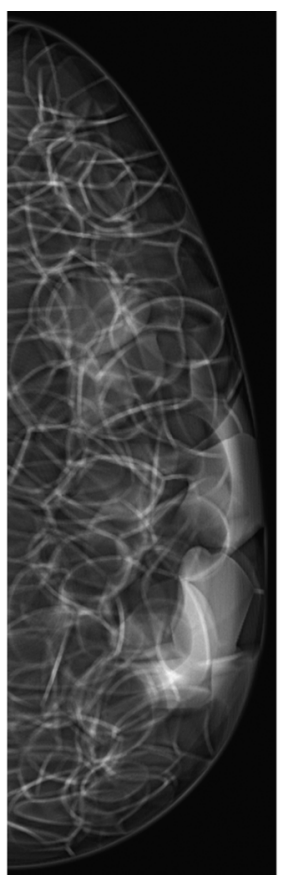

(a)

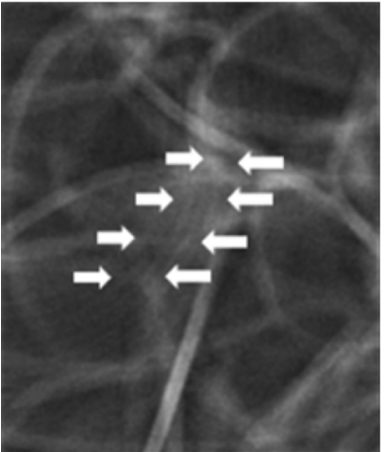

(d)

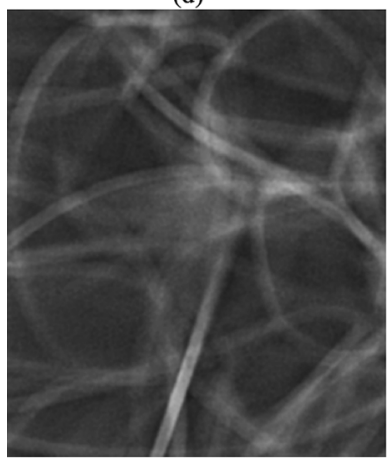

(f)

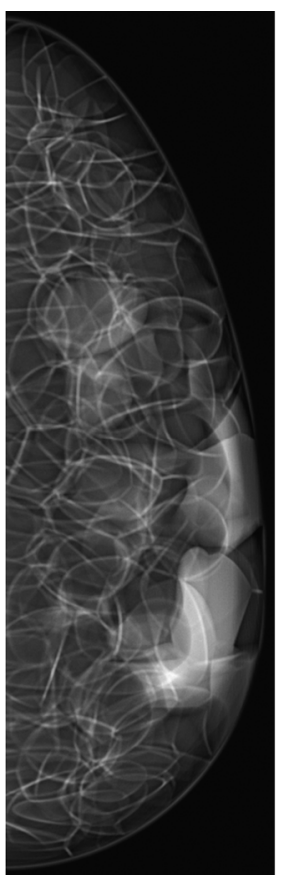

(b)

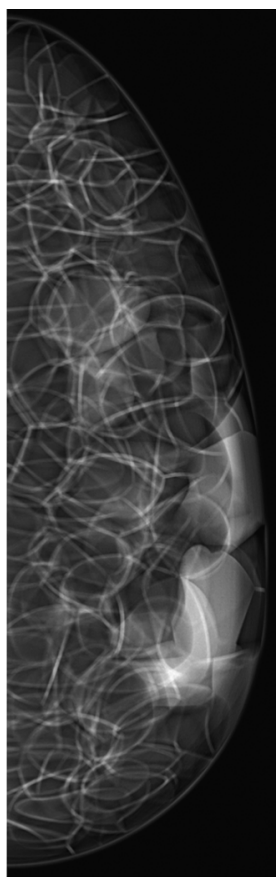

(c)

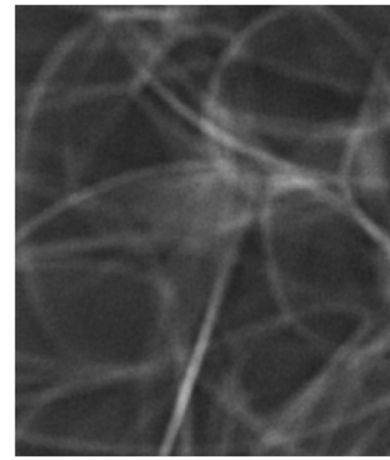

(e)

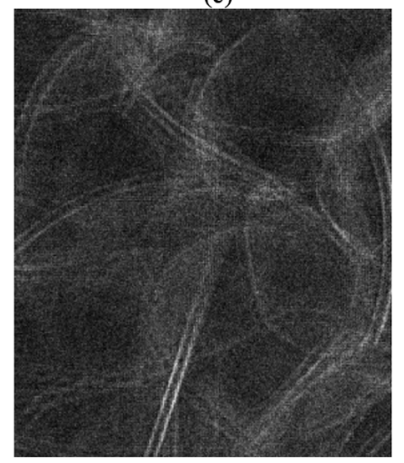

(g)

Fig. 9. Simulated projections of: (a) a phantom with $200 \mu \mathrm{m}$ voxels and no PV; (b) the phantom from (a) with simulated PV; (c) the same phantom generated at $100 \mu \mathrm{m}$ voxels and no PV; (d) A magnified detail from Fig. (a) (white arrows indicate stair-step quantization artifacts); (e) the corresponding detail from Fig. (b); (f) the corresponding detail of Fig. (c); (g) the difference between details in Figs. (f) and (e).

The number of points per voxel for the Monte Carlo simulation was varied $\left(\mathrm{N}_{\mathrm{MC}} \in\{63,500\}\right)$. The number of repetitions was $\mathrm{N}_{\text {repeat }}=100$. Table IV contains the $M S E_{A}$ for PV of skin, PV of ligaments in two material voxels and PV of ligaments in three material voxels, using the $200 \mu \mathrm{m}$ phantom 


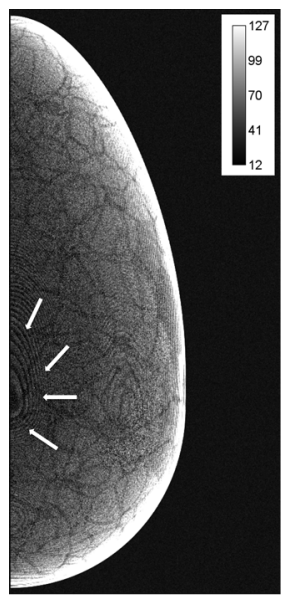

(a)

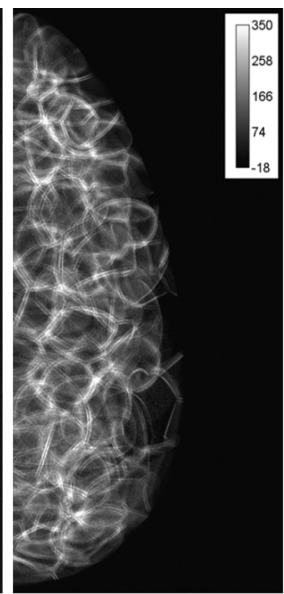

(b)

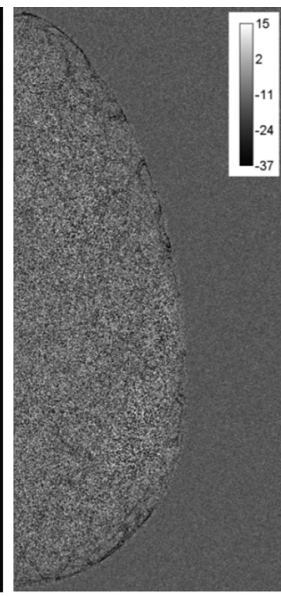

(c)
Fig. 10. Illustration of the effect of $\mathrm{PV}$ in simulated projections of phantoms. Shown are difference images emphasizing the contribution of (a) two-material voxels containing skin (cases 6-9 from Table II; white arrows indicate ripple artifacts); (b) two-material voxels containing ligaments (cases 10 and 11); and (c) three-material voxels (cases 12 and 13).

TABLE IV

$M S E_{A}, M S E_{M C}$ OBTAINED USING REPETITIONS, AND SAMPLE Means for Three Types of VoXels; Monte Carlo Method USES $\mathrm{N}_{\mathrm{MC}}=63$ POINTS PER PV VOXEL

\begin{tabular}{|c|c|c|c|c|}
\hline \multicolumn{2}{|c|}{ Estimation method } & $\begin{array}{c}\text { Two material: } \\
\text { skin }\end{array}$ & $\begin{array}{c}\text { Two material: } \\
\text { ligaments }\end{array}$ & $\begin{array}{c}\text { Three } \\
\text { material: } \\
\text { ligaments }\end{array}$ \\
\hline & $M S E_{M C S}$ & $1.80 \mathrm{e}-03$ & $1.77 \mathrm{e}-03$ & $1.57 \mathrm{e}-03$ \\
\hline & $M S E_{M C R}$ & $1.78 \mathrm{e}-03$ & $1.75 \mathrm{e}-03$ & $1.56 \mathrm{e}-03$ \\
\hline \multirow[b]{2}{*}{$M S E_{A}$} & Sample means & $2.01 \mathrm{e}-05$ & $4.32 \mathrm{e}-04$ & $2.93 \mathrm{e}-04$ \\
\hline & Repetition & $3.80 \mathrm{e}-05$ & $4.53 \mathrm{e}-04$ & $3.06 \mathrm{e}-04$ \\
\hline
\end{tabular}

TABLE V

THE Numbers of DifFERENT TyPes OF VOXELS AND THE AVERAGE EXECUTION TIMES PER PV VOXEL FOR THE PROPOSED METHOD AND FOR THE MONTE CARLO SIMULATION

\begin{tabular}{|c|c|c|c|c|}
\hline & $\begin{array}{l}\text { Voxel } \\
\text { type }\end{array}$ & $\begin{array}{l}\text { Two } \\
\text { material: } \\
\text { skin }\end{array}$ & $\begin{array}{l}\text { Two } \\
\text { material: } \\
\text { ligaments }\end{array}$ & $\begin{array}{c}\text { Three } \\
\text { materials }\end{array}$ \\
\hline & $\begin{array}{l}\text { Number } \\
\text { of Voxels }\end{array}$ & $1,597,042$ & $6,435,881$ & 87,610 \\
\hline \multirow{2}{*}{$\begin{array}{c}\text { Average } \\
\text { execution } \\
\text { time } \\
\text { per PV } \\
\text { voxel (ms) }\end{array}$} & $\begin{array}{c}\text { Linear } \\
\text { approximation }\end{array}$ & 0.556 & 0.380 & 4.75 \\
\hline & $\begin{array}{c}\text { Monte } \\
\text { Carlo }\left(\mathrm{N}_{\mathrm{MC}}=63\right)\end{array}$ & 0.371 & 21.6 & 22.1 \\
\hline
\end{tabular}

shown in Fig. 8. $M S E_{A}$ is computed using the Monte Carlo method with $\mathrm{N}_{\mathrm{MC}}=63$ random points, using both methods discussed in Section IV-A1. The corresponding estimated $M S E_{M C R}, M S E_{M C S}$ are also shown. Since we used $\mathrm{q}=6$ bits for representation of a partial volume percentage, the approximate quantization error, obtained using (21), was $M S E_{q}=2.09 \mathrm{e}-5$.

Table $\mathrm{V}$ lists the numbers of two-material voxels containing skin (cases 6-9; see Table II) and ligaments (cases 10 and 11), three-material voxels (cases 12 and 13), as well as the average execution times for one $\mathrm{PV}$ voxel using the linear approximation and the Monte Carlo method with $\mathrm{N}_{\mathrm{MC}}=63$. Note that total
TABLE VI

$M S E_{A}, M S E_{M C}$ Obtained Using the Sample Means Method for Three TyPes of Materials; Monte CARLo Method Uses $\mathrm{N}_{\mathrm{MC}}=500$ POINTS PER PV VOXEL

\begin{tabular}{cccc}
\hline \hline $\begin{array}{c}\text { Estimation } \\
\text { method }\end{array}$ & $\begin{array}{c}\text { Two material: } \\
\text { skin }\end{array}$ & $\begin{array}{c}\text { Two material: } \\
\text { ligaments }\end{array}$ & $\begin{array}{c}\text { Three } \\
\text { materials }\end{array}$ \\
\hline$M S E_{M C S}$ & $2.26 \mathrm{e}-04$ & $2.22 \mathrm{e}-04$ & $1.98 \mathrm{e}-04$ \\
$M S E_{A}$ & $3.60 \mathrm{e}-05$ & $4.48 \mathrm{e}-04$ & $2.92 \mathrm{e}-04$ \\
\hline \hline
\end{tabular}

TABLE VII

Values of the InPut Parameter Defining Relative Compartment Orientation, $\mathrm{R}_{0}$, AND the Relative Compartment Size, $\mathrm{R}_{s}$, Used FOR THE GENERATION OF THE Four ANALYZED Classes OF PHANTOMS

\begin{tabular}{|c|c|c|}
\hline $\begin{array}{ll} & \mathrm{r}_{s} \\
r_{0} & \\
\end{array}$ & 1 & {$[0.01,100]$} \\
\hline 1 & Class 1 & Class 2 \\
\hline$[0.25,4]$ & Class 3 & Class 4 \\
\hline
\end{tabular}

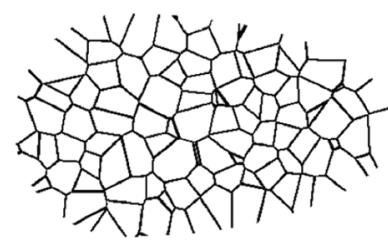

(a)

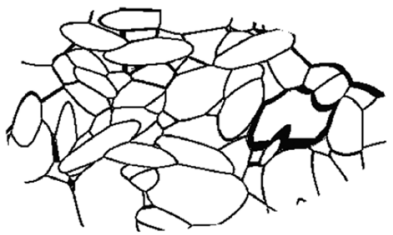

(c)

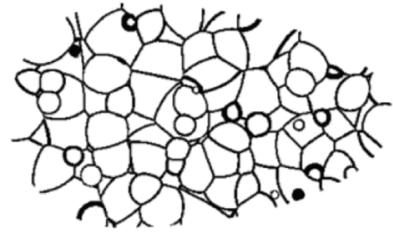

(b)

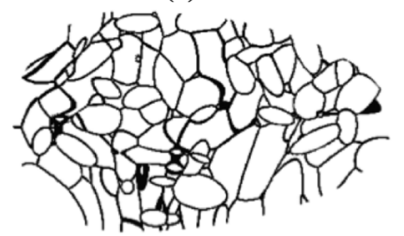

(d)
Fig. 11. Coronal cross-sections through sample phantoms from the four analyzed classes: (a) Class 1 (b) Class 2; (c) Class 3; (d) Class 4 (see Table VII).

execution time for the PV computation using the linear approximation, 3749s, was about 37 times less than with the Monte Carlo method $(\sim 141,000 \mathrm{~s})$. $\left(\mathrm{N}_{\mathrm{MC}}=63\right)$.

Table VI contains $M S E_{A}$ for PV of skin, PV of ligaments in two material voxels and PV of ligaments in three material voxels, on $200 \mu \mathrm{m}$ phantom shown in Fig. 8. $M S E_{A}$ is computed using the Monte Carlo method with $\mathrm{N}_{\mathrm{MC}}=500$ random points with the sample mean method. The corresponding estimated $M S E_{M C S}$ are also shown. Note that estimation of $M S E_{M C R}$ was not computationally feasible (the computation of $M S E_{M C R}$ would require excessively large computational time).

To determine the influence of ligament boundary non-linearity on the accuracy of the proposed PV estimation, we generated four $200 \mu \mathrm{m}$ phantoms corresponding to the classes discussed [49]. We varied the relative compartment orientation $\mathrm{r}_{0}$, and the relative compartment size, $\mathrm{r}_{s}$, as shown in Table VII.

Table VIII contains $M S E_{A}, M S E_{M C}$ obtained using the sample means method for three types of materials on four phantoms with different non-linearity of ligament boundaries specified by different ranges of $\mathrm{r}_{s}$ and $\mathrm{r}_{0}$ (See Fig. 11); Monte Carlo method uses $\mathrm{N}_{\mathrm{MC}}=63$ points per $\mathrm{PV}$ voxel. 
TABLE VIII

$M S E_{A}, M S E_{M C}$ FOR FoUR PHANTOMS With DIFFERENT NON-LINEARITY OF LIGAMENT BOUNDARIES SPECIFIED BY DIFFERENT RANGES OF $\mathbf{R}_{s}$ AND $\mathrm{R}_{0}$. The Monte Carlo Method Uses $\mathrm{N}_{\mathrm{MC}}=63$ Points Per PV Voxel. A) $\mathrm{r}_{s}=1, \mathrm{r}_{0}=1$ B) $\mathrm{r}_{s}$ in $\left.[0.01,100], \mathrm{r}_{0}=1 \mathrm{C}\right) \mathrm{r}_{s}=1, \mathrm{r}_{0}$ in $[0.25,4]$ D) $\mathrm{r}_{s}$ in $[0.01,100], \mathrm{r}_{0}$ in $[0.25,4]$

\begin{tabular}{|c|c|c|c|c|}
\hline \hline $\begin{array}{c}\text { Class } \\
1\end{array}$ & $\begin{array}{c}\text { Estimation } \\
\text { method }\end{array}$ & $\begin{array}{c}\text { Two material: } \\
\text { Skin }\end{array}$ & $\begin{array}{c}\text { Two material: } \\
\text { ligaments }\end{array}$ & $\begin{array}{c}\text { Three } \\
\text { materials }\end{array}$ \\
\hline \multicolumn{2}{|c|}{$M S E_{M C S}$} & $1.80 \mathrm{e}-03$ & $1.76 \mathrm{e}-03$ & $1.59 \mathrm{e}-03$ \\
\hline \multicolumn{2}{|c|}{$M S E_{A}$} & $1.98 \mathrm{e}-05$ & $4.14 \mathrm{e}-05$ & $2.57 \mathrm{e}-05$ \\
\hline \hline
\end{tabular}

a)

\begin{tabular}{|c|c|c|c|c|}
\hline \hline $\begin{array}{c}\text { Class } \\
2\end{array}$ & $\begin{array}{c}\text { Estimation } \\
\text { method }\end{array}$ & $\begin{array}{c}\text { Two material: } \\
\text { Skin }\end{array}$ & $\begin{array}{c}\text { Two material: } \\
\text { ligaments }\end{array}$ & $\begin{array}{c}\text { Three } \\
\text { materials }\end{array}$ \\
\hline \multicolumn{2}{|c|}{$M S E_{M C S}$} & $1.80 \mathrm{e}-03$ & $1.75 \mathrm{e}-03$ & $1.57 \mathrm{e}-03$ \\
\hline \multicolumn{2}{|c|}{$M S E_{A}$} & $2.30 \mathrm{e}-05$ & $3.70 \mathrm{e}-04$ & $1.16 \mathrm{e}-04$ \\
\hline \hline
\end{tabular}

b)

\begin{tabular}{|c|c|c|c|c|}
\hline \hline $\begin{array}{c}\text { Class } \\
3\end{array}$ & $\begin{array}{c}\text { Estimation } \\
\text { method }\end{array}$ & $\begin{array}{c}\text { Two material: } \\
\text { Skin }\end{array}$ & $\begin{array}{c}\text { Two material: } \\
\text { ligaments }\end{array}$ & $\begin{array}{c}\text { Three } \\
\text { materials }\end{array}$ \\
\hline \multicolumn{2}{|c|}{$M S E_{M C S}$} & $1.80 \mathrm{e}-03$ & $1.81 \mathrm{e}-03$ & $1.59 \mathrm{e}-03$ \\
\hline \multicolumn{2}{|c|}{$M S E_{A}$} & $1.53 \mathrm{e}-05$ & $1.04 \mathrm{e}-03$ & $3.30 \mathrm{e}-04$ \\
\hline \hline
\end{tabular}

c)

\begin{tabular}{|c|c|c|c|c|}
\hline \hline $\begin{array}{c}\text { Class } \\
4\end{array}$ & $\begin{array}{c}\text { Estimation } \\
\text { method }\end{array}$ & $\begin{array}{c}\text { Two material: } \\
\text { Skin }\end{array}$ & $\begin{array}{c}\text { Two material: } \\
\text { ligaments }\end{array}$ & $\begin{array}{c}\text { Three } \\
\text { materials }\end{array}$ \\
\hline \multicolumn{2}{|c|}{$M S E_{M C S}$} & $1.80 \mathrm{e}-03$ & $1.79 \mathrm{e}-03$ & $1.58 \mathrm{e}-03$ \\
\hline \multicolumn{2}{|c|}{$M S E_{A}$} & $2.17 \mathrm{e}-05$ & $2.14 \mathrm{e}-03$ & $5.39 \mathrm{e}-04$ \\
\hline \hline
\end{tabular}

d)

\section{DISCUSSION}

Fig. 8 suggests that the simulation of all 13 cases of PV voxels is qualitatively correct. The voxels containing two materials are detected at the boundaries of two materials (e.g., skin, compartment). The three material voxels are detected where the skin meets Cooper's ligaments and a compartment. Computed percentages (PVs) of skin and ligaments gradually decrease when departing from the inside of the skin (ligaments) (Figs. 8(b), 8(c)).

In Fig. 9(e), for a phantom with simulated PV, the equivalent $\mathrm{x}$-ray attenuations of voxels on skin/air and ligaments/fat tissue boundaries were lower than the x-ray attenuation of dense tissue, hence the quantization artifacts were reduced and Cooper's ligaments and skin appeared thinner and their boundaries smoother in the projection (as compared to the phantom without PV, Fig. 9(d)). This is confirmed by the difference between projections of phantoms with two material PV voxels and with PV voxels containing skin (Fig. 10(b)).

In Fig. 9(e), the characteristic stair-step quantization artifacts on tissue boundaries were noticeably reduced with simulated PV. Simulation of two-material voxels with skin leads to reduction of ripple artifacts due to sudden change of attenuation at the skin boundary. (Fig. 10(a)). Note that here we represent linear x-ray attenuation coefficient of a PV voxel as a weighted average of the attenuation coefficients of materials contained in the voxel (instead of using a single material attenuation coefficient). Hence, the proposed method can reduce aliasing due to improved sampling of a continuous phantom. Comparison of Figs. 9(e) and 9(f) indicates similar appearance of a phantom with PV simulated at a larger voxel size $(200 \mu \mathrm{m})$ to a phantom simulated at a smaller voxel size $(100 \mu \mathrm{m})$ with no simulated PV. Note that a Cooper's ligament in the lower central portion of Fig. 9(e) with simulated PV appears thinner, even when compared with a smaller voxel size phantom without PV (Fig. 9(f)). This is also notable from Fig. $9(\mathrm{~g})$. Hence, the application of PV may lead to an improvement in image quality without reducing voxel size. In comparison to noticeable quality improvement of simulation of two-material PV voxels, the simulation of three-material voxels leads to a relatively smaller improvement in image quality, by removing high-frequency artifacts (see Fig. 10(c)).

The estimated accuracy of the PV computation $\left(M S E_{A}\right)$ is better when using the proposed approximation, than using the MC with 63 points, as indicated in Table IV. For skin, the accuracy of approximation is close to the approximate quantization error $M S E_{q}$ (calculated in the beginning of Section V-B). The statistically insignificant discrepancy (2.09e-5 vs. 2.01e-5) could be explained by error in estimating $M S E_{M C}$. Note that using $\mathrm{N}_{\mathrm{MC}}=63$ points per voxel in Monte Carlo estimation corresponds to 6-bits resolution of the obtained PV estimates-the same as the resolution due to discretization of the approximation.

Comparison of Tables IV and VI shows that the estimate of $M S E_{A}$ is stable (i.e., does not change much) with increasing $\mathrm{N}_{\mathrm{MC}}$. Hence, the use in practice of relatively small $\mathrm{N}_{\mathrm{MC}}(=63)$ to estimate $M S E_{A}$ is justified. When $\mathrm{N}_{\mathrm{MC}}$ was increased, $M S E_{M C}$ decreased (as expected from (29)) and became comparable to $M S E_{A}$ on two-material and three-material ligament voxels. Observe, however, that this comparable accuracy is achieved at the expense of additional computational time. Hence, our proposed approximation method is preferable for both fast and accurate estimation of PV.

For a phantom with very linear ligament boundaries (Fig. 11(a)), $M S E_{A}$ is very close to $M S E_{q}$ (see Table VIII(a)). In this case, the linear approximation clearly has better accuracy than $\mathrm{MC}$ with 63 random points. As we can see, when $\mathrm{r}_{0}=1$ but relative compartment sizes vary in $[0.01,100]$ (Fig. 11(b)), the boundaries of ligaments are non-linear but relatively smooth. Hence, the MSE of the linear approximation of twoor three-material ligaments are smaller than for the MSE of the Monte Carlo (see Table VIII(b)). Fig. 11(c) shows that when $\mathrm{r}_{0}$ is allowed to vary in $[0.25,4]$ but relative compartment size is $\mathrm{r}_{s}=1$, the phantom's ligament boundaries are less linear. As a consequence, $M S E_{A}$ for ligament voxels are larger than in the previous case (Figs. 11(a) and 11(b)). Nevertheless, linear approximation still has smaller error than MC. Fig. 11(d) shows that when $\mathrm{r}_{0}$ vary in $[0.25,4]$ but relative compartment sizes $\mathrm{r}_{s}$ vary in $[0.01,100]$, ligament boundaries are very non-linear. As a result, now $M S E_{A}$ for ligaments is larger than $M S E_{M C}$. In contrast, the volumes of three-material voxels are bounded by relatively smooth skin surface (in addition to non-linear ligament surface), and in such cases linear approximation outperforms MC.

Our algorithms for two and three materials are very efficient. In the two-material case, Algorithm A1 can be used to compute partial volume by solving one inner product and the volume of a few geometric primitives. In the three-material case, Algorithm $\mathrm{A} 3$ has converted the $3 \mathrm{D}$ volume problem into a $2 \mathrm{D}$ area 
problem using the Gauss-Ostrogradsky theorem. Moreover, Algorithm A3 can be used for any number of materials, but for two-material cases, Algorithm A1 is faster.

The obtained average execution times of PV estimation per voxel, Table $\mathrm{V}$, are platform and implementation dependent. Using the Monte Carlo method for two-material voxels containing skin (cases 6-9) is relatively straightforward resulting in smaller average execution times than using our implementation of the proposed algorithm. Note that while being conceptually simple, estimation using the Monte Carlo method of voxels containing ligament tissue relies upon computation of distance from the median surface which is resource intensive (it reduces to numerical solution of the polynomial equation of 6-th degree [44]). Since PV with two-materials containing ligaments (cases 10-11) are predominant in the considered phantom, the total time to compute partial volumes using the linear approximation was much smaller than using the Monte Carlo method with $\mathrm{N}_{\mathrm{MC}}=63$ points. Therefore, using the linear approximation should be faster than the Monte Carlo method. On the other hand, unlike the linear approximation, where the accuracy depends on the linearity of the material boundaries, the accuracy of the Monte Carlo method can be controlled (by choosing large enough $\mathrm{N}_{\mathrm{MC}}$, see (29)). Hence, if the accurate computation of partial volumes separated by highly non-linear surfaces is necessary, or if the application/platform (e.g., parallel platforms) where the Monte Carlo method is efficient are available, the Monte Carlo may be a method of choice for computing partial volumes. The determination of smallest $\mathrm{N}_{\mathrm{MC}}$, for a given $M S E_{M C}$ is part of our work in progress.

For realistic cases of non-linear ligament boundaries, the estimated $M S E_{A}$ of two-material voxels containing ligaments and of three-material voxels were larger than the quantization error $M S E_{q}$ when $\mathrm{q}=6$ bits are used. Hence, it is sufficient to use 6 bits to represent partial volumes computed using the proposed linear approximation. Note that in [38] we proposed using 7 bits per partial volume.

Note, finally, that we have proposed two techniques to estimate $M S E_{M C}$, and experimentally confirmed their similar behavior in the proposed application. The second technique is based upon the estimation of sample means of computed $\mathrm{PV}_{\mathrm{MC}}$, which avoids the estimation of $\varepsilon_{M C}$. This technique does not require knowledge of true values of $\mathrm{PV}_{j}$ at each voxel nor the availability of distribution $p(\mathrm{PV})$, and could be thus potentially used in other estimation problems.

\section{A. Limitations}

The proposed method utilizes a linear approximation of the bounding surfaces between simulated materials. A better approximation (e.g., quadratic; piecewise linear) may still be needed if surfaces separating different materials have large curvature. If the boundaries are highly non-linear, computation of the PV effect using the Monte Carlo (MC) method may be a better choice (since MC provides a controllable approximation error), provided fast enough hardware/implementation. The proposed encoding scheme reserves four bits to represent the material type. As so far we have utilized 2 bits only, more material types (e.g., lesions; calcifications; ducts) may be represented.

\section{ALGORITHM A1}

Conceptual algorithm for computing partial volume of a voxel above a given plane

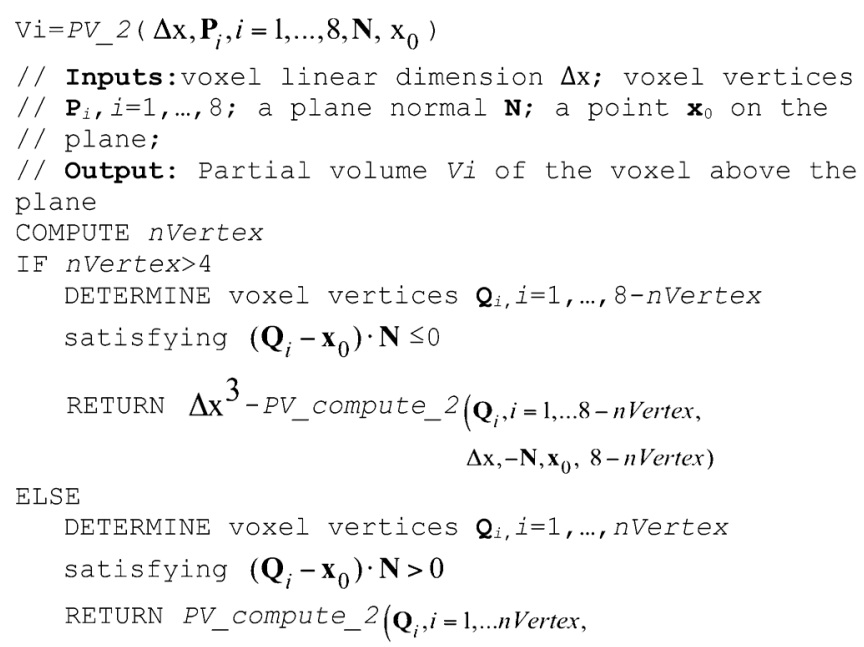

$$
\left.\Delta \mathrm{x}, \mathbf{N}, \mathbf{x}_{0}, n \text { Vertex }\right)
$$

Difference between projections of a lower voxel resolution PV phantom and a higher resolution non-PV phantom may include "gauze-like" ringing artifacts as seen in Fig. 9(g). Characterization of the artifacts and investigation of their cause are ongoing. With a large parallel computer system, it is feasible to model the different tissue types by using the voxels with smaller size $(100 \mu \mathrm{m}, 70 \mu \mathrm{m})$ where every voxel will be a separate tissue type [52], [53]. However, application of the proposed method would still lead to further improvement of accuracy of these phantoms. The proposed algorithm could be advantageous at lower voxel resolution for reducing the required storage space and faster data transfer and analysis. Selection of the optimal voxel size is a topic of the ongoing discussions in the AAPM TG234 on the Virtual Tools for the Validation of Novel 3D/4D Breast Imaging Systems.

\section{CONCLUSIONS}

We have developed a method for simulating PV in software breast phantom voxels, which contains multiple simulated tissues. The percentage of simulated tissues was estimated using a planar approximation of the boundary between different tissue regions, based upon the segmentation into geometric primitives and the Gauss-Ostrogradsky theorem. A quantitative assessment of the planar approximation using Monte Carlo estimation of computed PV showed satisfactory accuracy of the proposed method. A qualitative comparison of simulated mammographic projections confirmed that the PV simulation can improve the image quality by reducing the quantization artifacts. A future work would involve human or model observer studies to quantify the image quality improvement.

\section{APPENDIX}

\section{A. Pseudocode of Algorithms for Computing Partial Volume of a Voxel Above a Plane and Above two Planes}

See Algorithms A1-A3. 


\section{ALGORITHM A2}

Algorithm for computation of partial volume of a voxel above a plane for different number of vertices above the plane

$\mathrm{Vi}=P V_{\text {_compute_}} 2\left(\mathbf{Q}_{i}, i=1, \ldots n\right.$ Vertex $, \Delta \mathbf{x}, \mathbf{N}, \mathbf{x}_{0}$, nVertex $)$

// Inputs: Voxel vertices $\mathbf{Q}_{i}, i=1, \ldots$, nVertex above

// plane $\pi$ (nVertex $\leq 4$ !)

$/ /$ voxel linear dimension $\Delta x$

$/ /$ normal $\mathbf{N}$ and a point $\mathbf{x}_{0}$ specifying plane $\boldsymbol{\pi}$.

// Output: Partial volume Vi of the voxel above the // plane $\pi$

IF $\quad$ nVerte $x==0$

RETURN 0. //Partial volume is 0

ELSEIF nVertex $==1$

//Volume is a right angle triangular pyramid.

//CASE A (see Table 3)

COMPUTE nPoint $=2 \star n$ Vertex +1 .

//the number of intersections.

COMPUTE intersections $\mathbf{P}, \mathbf{Q}, \mathbf{R}$ between $\boldsymbol{\pi}$ and

$e_{i}$, the edges containing $\mathbf{Q}_{1}$.

COMPUTE the distances between intersections and $\mathbf{Q}_{1}$.

RETURN volume of a tetrahedron $\mathbf{P Q R}_{1}$.

// (See Fig. $4(a)$ )

ELSEIF nVertex==2

DETERMINE the edge e containing $\boldsymbol{Q}_{1}$ and $\boldsymbol{Q}_{2}$

IF $\mathbf{N} \perp e$

//Volume is a triangular prism. CASE B1

COMPUTE intersections $\mathbf{P}, \mathbf{Q}$ between $\boldsymbol{\pi}$ and edges containing $\mathbf{Q}_{1}$ (except e).

RETURN volume of a prism defined with base $\mathbf{P Q Q}_{1}$ and height $e$.

ELSE //(See Fig. 4(b))

//Volume is a triangular cut pyramid. CASE B2 COMPUTE $n$ Point $=2{ }^{*} n$ Vertex +1 .

$/ /$ the number of intersections.

COMPUTE intersections $\mathbf{P}, \mathbf{Q}, \mathbf{R}, \mathbf{S}, \mathbf{T}$

between $\pi$ and each edge (or extension of edge) containing $\boldsymbol{Q}_{1}$ or $\boldsymbol{Q}_{2}$.

RETURN volume difference $\mathrm{b} / \mathrm{w}$ tetrahedra

PQRQ $_{1}$, PSTQ $_{2}$. //See Fig. 4 (C) ENDIF

ELSEIF nVertex $==3$

//Volume is a "double cut" pyramid. CASE C COMPUTE nPoint=2*nVertex+1.

COMPUTE intersections $\mathbf{P}, \mathbf{Q}, \mathbf{R}, \mathbf{S}, \mathbf{T}, \mathbf{U}, \mathbf{W}$ between $\boldsymbol{\pi}$ and each edge (or extension of edge) containing $\boldsymbol{Q}_{1}, \mathbf{Q}_{2}$ or $\boldsymbol{Q}_{3}$.

RETURN volume difference $\mathrm{b} / \mathrm{w}$ tetrahedra $\mathbf{P Q R}_{1}$, PSTQ $_{2}$, QUWQ $_{3}$. //See Fig.4(d)

ELSEIF nVertex $==4$

IF vertices $\boldsymbol{Q}_{i} i=1, \ldots, 4$ are coplanar

//Volume is a prismoid. CASE D1

COMPUTE intersections $P, Q, R, S$ between $\pi$ and edges vertical to plane defined by $\boldsymbol{Q}$ $i=1, \ldots, 4$.

RETURN volume of prismoid $\mathbf{Q}_{1} \mathbf{Q}_{2} \mathbf{Q}_{3} \mathbf{Q}_{4}$ PQRS .

ELSE //See Fig.4(e)

//Volume is a "triple cut" pyramid.

//CASE D2

COMPUTE nPoint=2*nVertex 1 . COMPUTE intersections $\mathbf{P}, \mathbf{Q}, \mathbf{R}, \mathbf{S}, \mathbf{T}, \mathrm{U}$, $\mathbf{W}, \mathbf{Y}, \mathbf{Z}$ between $\pi$ and each edge (or extension of edge) containing $\mathbf{Q}_{1}, \mathbf{Q}_{2}, \mathbf{Q}_{3}$ or $\mathbf{Q}_{4}$

RETURN volume difference $\mathrm{b} / \mathrm{w}$ tetrahedron $\mathbf{P Q R Q}_{2}$ and tetrahedra $\mathbf{P S T Q _ { 1 }}, \mathbf{Q U W Q _ { 3 }}$, and $\mathrm{RYZQ}_{4}$.

ENDIF
ALGORITHM A3

Algorithm for computation of partial volume of a voxel above two planes

$\mathrm{V} i=P V_{-} 3\left(\Delta \mathbf{x}, \mathbf{P}_{i}, i=1, \ldots, 8, \mathbf{x}_{1}, \mathbf{N}_{1}, \mathbf{x}_{2}, \mathbf{N}_{2}\right)$

// Inputs: voxel linear dimension $\Delta \mathrm{x}$;

// voxel vertices $\mathbf{P}_{i}, i=1, \ldots, 8$

// plane normals $\mathbf{N}_{1}, \mathbf{N}_{2}$ of planes $\boldsymbol{\pi}_{1}, \boldsymbol{\pi}_{2}$; points $\mathbf{x}_{1}, \mathbf{x}_{2}$

// on the planes

// Output: Partial volume Vi of the voxel above the // planes $\pi_{1}, \pi_{2}$

COMPUTE nVertex as the number of vertices $\mathbf{P}_{i}$

satisfying $\left(\mathbf{P}_{i}-\mathbf{x}_{1}\right) \cdot \mathbf{N}_{1}>0$ and $\left(\mathbf{P}_{i}-\mathbf{x}_{2}\right) \cdot \mathbf{N}_{2}>0$.

IF $\quad n$ Vertex $==8$

RETURN $\Delta \mathrm{x}^{3}$

ELSEIE nVertex $=0$ RETURN PV_compute_3 $\left(\Delta \mathbf{x}, \mathbf{x}_{1}, \mathbf{N}_{1}, \mathbf{x}_{2}, \mathbf{N}_{2}, \mathbf{P}_{i}, i=1, \ldots, 8\right)$.

ELSE

COMPUTE nVertex1 and nVertex2, the number of vertices above $\boldsymbol{\pi}_{1}$ and $\boldsymbol{\pi}_{2}$. IF nVertex $1^{*}$ nVertex $2 \sim=0$

//there is no vertex above both planes, but

//there is a vertex above each plane.

RETURN PV_2( $\left.\Delta \mathrm{x}, \mathbf{P}_{i}, i=1, \ldots, 8, \mathbf{N}_{1}, \mathbf{x}_{1}\right)-P V$ compute_3(

$\left.\Delta \mathrm{x}, \mathbf{x}_{1}, \mathbf{N}_{1}, \mathbf{x}_{2},-\mathbf{N}_{2}, \mathbf{P}_{i}, i=1, \ldots, 8\right)$. //(See Fig. 7)

ELSE

RETURN 0

ENDIF ENDIF

Vi=PV_compute_3( $\left.\Delta \mathbf{x}, \mathbf{x}_{1}, \mathbf{N}_{1}, \mathbf{x}_{2}, \mathbf{N}_{2}, \mathbf{P}_{i}, i=1, \ldots, 8\right)$

// Inputs: voxel linear dimension $\Delta \mathrm{x}$;

// plane normals $\mathbf{N}_{1}, \mathbf{N}_{2}$ of planes $\boldsymbol{\pi}_{1}, \boldsymbol{\pi}_{2}$; points $\mathbf{x}_{1}, \mathbf{x}_{2}$

// on the planes

// Voxel vertices $\mathbf{P}_{i}, i=1, \ldots, 8$ such that at least one vertex is above both planes $\boldsymbol{\pi}_{1}, \boldsymbol{\pi}_{2}$

// Output: Partial volume Vi of the voxel above the // planes $\pi_{1}, \pi_{2}$

CHOOSE any vertex of the voxel above the two planes, denoted by $\mathbf{Q}_{1}$.

FOR each voxel side $\sigma_{i}, i=1, \ldots, 8$ that does not contain $\mathrm{Q}$

DETERMINE the set $S_{\sigma_{i}}$ of points above or on $\boldsymbol{\pi}_{1}$ and $\boldsymbol{\pi}_{2}$ belonging to one of the following sets: intersections between edges of $\sigma_{i}$ and planes $\boldsymbol{\pi}_{1}, \boldsymbol{\pi}_{2}$; intersections between $\boldsymbol{\pi}_{1}, \boldsymbol{\pi}_{2}$ and $\sigma_{i}$; vertices belonging to $\sigma_{i}$.

COMPUTE $S_{i}$ as the area of convex polygon with vertices from $S_{\sigma_{i}}$.

COMPUTE the distances $d_{j}$ between $\boldsymbol{Q}_{1}$ and $\boldsymbol{\pi}_{j}, j=1,2$.

FOR each plane $\boldsymbol{\pi}_{j}, j=1,2$

DETERMINE the set $S_{\pi_{j}}$ belonging to one of the

following sets: intersections between $\boldsymbol{\pi}_{j}$ and the voxel edges that are above or on

$\boldsymbol{\pi}_{\mathrm{k}}, k \neq j$; intersections of $\boldsymbol{\pi}_{1}, \boldsymbol{\pi}_{2}$ and the surface of the voxel.

COMPUTE $A_{j}$ as the area of a convex polygon with vertices from $S_{\pi_{i}}$.

RETURN Vi $=\frac{1}{3}\left[\left(S_{1}+S_{2}+S_{3}\right) \Delta \mathrm{x}+A_{1} d_{1}+A_{2} d_{2}\right]$

\section{B. Some Properties of Monte Carlo Estimation of Partial Volume}

Observe that the true value of a partial volume PV is the probability that a randomly chosen point during the Monte Carlo 
computation of partial volume is within the volume of interest. Hence, the count of points $\left(\mathrm{N}_{\mathrm{MCI}}\right.$ in (16)) follows a Binomial distribution with expectation $\mathrm{N}_{\mathrm{MC}} \cdot \mathrm{PV}$ and variance $\mathrm{N}_{\mathrm{MC}} \cdot \mathrm{PV}$. $(1-\mathrm{PV})$ [43]. From this and (16), (17), follows:

$$
\begin{aligned}
& \mathrm{E}\left(\varepsilon_{M C} \mid \mathrm{PV}\right)=0 \\
& \mathrm{E}\left(\varepsilon_{M C}^{2} \mid \mathrm{PV}\right)=\frac{\mathrm{PV}(1-\mathrm{PV})}{\mathrm{N}_{\mathrm{MC}}} .
\end{aligned}
$$

Note that it is suitable to treat the true value of the partial volume, $\mathrm{PV}$, as a random variable (since it varies throughout the phantom in fashion unknown to the algorithm for PV estimation). Using the conditional expectation $\mathrm{E}\left(\varepsilon_{M C}^{2} \mid \mathrm{PV}\right)$, the expectation $E\left(\varepsilon_{M C}^{2}\right)$ of the error of the Monte Carlo method can be expressed as [43]:

$$
E\left(\varepsilon_{M C}^{2}\right)=\int_{0}^{1} E\left(\varepsilon_{M C}^{2} \mid \mathrm{PV}\right) \cdot p(\mathrm{PV}) d \mathrm{PV}
$$

By combining (A2) and (A3) we can easily obtain:

$$
\begin{aligned}
E\left(\varepsilon_{M C}^{2}\right) & =\int_{0}^{1} \frac{\mathrm{PV}(1-\mathrm{PV})}{\mathrm{N}_{\mathrm{MC}}} \cdot p(\mathrm{PV}) d \mathrm{PV} \\
& =\frac{1}{\mathrm{~N}_{\mathrm{MC}}}\left(E(\mathrm{PV})-E\left(\mathrm{PV}^{2}\right)\right) .
\end{aligned}
$$

Note that $E\left(\varepsilon_{M C}^{2}\right)$ depends on the distribution of PV and hence it may depend on a partial volume case (see Section III-B).

Also, similarly, using (A1) we can obtain:

$$
\begin{aligned}
E\left(\varepsilon_{M C} \cdot \mathrm{PV}\right) & =\int_{0}^{1} E\left(\varepsilon_{M C} \mathrm{PV}\right) \cdot \mathrm{PV} \cdot p(\mathrm{PV}) d \mathrm{PV}=0 \\
E\left(\varepsilon_{M C}\right) & =\int_{0}^{1} E\left(\varepsilon_{M C} \mid \mathrm{PV}\right) \cdot p(\mathrm{PV}) d \mathrm{PV}=0
\end{aligned}
$$

Note that $E\left(\varepsilon_{M C}\right)$ does not depend on the distribution of $\mathrm{PV}$ and hence does not depend on a partial volume case (see Section III-B).

Due to (A6),

$$
\mathrm{E}(\mathrm{PV})=\mathrm{E}\left(\mathrm{PV}_{\mathrm{MC}}\right)
$$

Note that $\mathrm{PV}_{\mathrm{A}}$ is a function of $\mathrm{PV}$ (in the ideal case, $\mathrm{PV}_{\mathrm{A}}=$ $\mathrm{PV}$ !) and therefore $\varepsilon_{A}=\varepsilon_{A}(\mathrm{PV})$. Due to (A1):

$$
E\left(\varepsilon_{M C} \varepsilon_{A}\right)=\int E\left(\varepsilon_{M C} \mid \mathrm{PV}\right) \cdot \varepsilon_{A}(\mathrm{PV}) d \mathrm{PV}=0,
$$

i.e., the approximation error and the error of Monte Carlo method are not correlated. Note that (A8) holds for linear and non-linear approximation methods.

\section{ACKNOWLEDGMENT}

The authors are thankful to Ms. Susan Ng from Real-Time Tomography (Villanova, PA) for processing the simulated projection images. Also, the authors wish to thank the anonymous reviewers whose suggestions significantly improved the quality of the manuscript.

\section{REFERENCES}

[1] P. Taylor and R. Owens, "Simulated mammography using synthetic 3D breasts," in Proc. 4th Int. Workshop Digital Mammogr., 1998, pp. 283-290.
[2] P. R. Bakic, M. Albert, D. Brzakovic, and A. D. A. Maidment, "Mammogram synthesis using a 3D simulation. I. Breast tissue model and image acquisition simulation," Med. Phys., vol. 29, no. 9, pp. 2131-2139, 2002

[3] P. R. Bakic, M. Albert, D. Brzakovic, and A. D. A. Maidment, "Mammogram synthesis using a 3D simulation. II. Evaluation of synthetic mammogram texture," Med. Phys., vol. 29, no. 9, pp. 2140-2151, 2002.

[4] P. R. Bakic, M. Albert, D. Brzakovic, and A. D. A. Maidment, "Mammogram synthesis using a three-dimensional simulation. III. Modeling and evaluation of the breast ductal network," Med. Phys., vol. 30, no. 7, pp. 1914-1925, 2003.

[5] K. Bliznakova, Z. Bliznakov, V. Bravou, Z. Kolitsi, and N. Pallikarakis, "A three-dimensional breast software phantom for mammography simulation," Phys. Med. Biol., vol. 48, no. 22, pp. 3699-3719, 2003.

[6] C. Zhang, P. R. Bakic, and A. D. A. Maidment, "Development of an anthropomorphic breast software phantom based on region growing algorithm," in Proc. SPIE 6918, Med. Imag., Visualizat., Image Guided Procedures, Model., 2008, p. 69180V.

[7] K. Bliznakova, S. Suryanarayanan, A. Karellas, and N. Paiilikarakis, "Evaluation of an improved algorithm for producing realistic 3D breast software phantoms: Application for mammography," Med. Phys., vol. 37, no. 11 , pp. 5604-5617, 2010.

[8] I. Reiser, S. Lee, K. Little, and R. M. Nishikawa, "Toward validation of a 3D structured background model for breast imaging," in Proc. SPIE 7627, Med. Imag., Image Perception, Observer Performance, Technol. Assessment, 2008, p. 762716.

[9] B. Chen et al., "An anthropomorphic breast model for breast imaging simulation and optimization," Acad. Radiol., vol. 18, no. 5, pp. 536-546, 2011.

[10] A. B. Lau, I. Reiser, R. M. Nishikawa, and P. R. Bakic, "A statistically defined anthropomorphic software breast phantom," Med. Phys., vol. 39, no. 6, pp. 3375-3385, 2012.

[11] D. D. Pokrajac, A. D. A. Maidment, and P. R. Bakic, "Optimized generation of high resolution breast anthropomorphic software phantoms," Med. Phys., vol. 39, no. 4, pp. 2290-3302, 2012.

[12] C. Hoeschen et al., "A high resolution voxel phantom of the breast for dose calculations in mammography," Radiat. Protection Dosimetry, vol. 114, no. 1-3, pp. 406-409, 2005.

[13] J. M. O'Connor, M. Das, C. Didier, M. Mah'D, and S. J. Glick, “Comparison of two methods to develop breast models for simulation of breast tomosynthesis and CT," in Proc. 9th Int. Workshop Digital Mammogr., 2008, pp. 417-425.

[14] C. M. Li, W. P. Segars, G. D. Tourassi, J. M. Boone, and J. T. Dobbins III, "Methodology for generating a 3D computerized breast phantom from empirical data," Med. Phys., vol. 36, no. 7, pp. 3122-3131, 2009.

[15] J. M. O'Connor, M. Das, C. Didier, M. Mah'D, and S. J. Glick, "Development of an ensemble of digital breast object models," in Proc. 10th Int. Workshop Digital Mammogr., 2010, pp. 54-61.

[16] N. Kiarashi et al., "Development of matched virtual and physical breast phantoms based on patient data," in Proc. SPIE 8668, Med. Imag., Phys. Med. Imag., 2013, p. 866805.

[17] J. M. O'Connor, M. Das, C. S. Didier, M. Mahd, and S. J. Glick, "Generation of voxelized breast phantoms from surgical mastectomy specimens," Med. Phys., vol. 40, no. 4, p. 041915, 2013.

[18] P. R. Bakic et al., "Validation and optimization of digital breast tomosynthesis reconstruction using an anthropomorphic software breast phantom," in Proc. SPIE 7622, Med. Imag., Phys. Med. Imag., 2010, p. $76220 \mathrm{~F}$.

[19] P. R. Bakic, P. Ringer, J. Kuo, S. Ng, and A. D. A. Maidment, "Analysis of geometric accuracy in digital breast tomosynthesis reconstruction," in Proc. 10th Int. Workshop Digital Mammogr., 2010, pp. 62-69.

[20] R. Zeng, S. Park, P. R. Bakic, and K. J. Myers, "Is the outcome of optimizing the system acquisition parameters sensitive to the reconstruction algorithm in digital breast tomosynthesis?," in Proc. 11th Int. Workshop Breast Imag., 2012, pp. 346-353.

[21] M. A. C. Vieira, P. R. Bakic, and A. D. A. Maidment, "Effect of denoising on the quality of reconstructed images in digital breast tomosynthesis," Proc. SPIE 8668, Med. Imag., Phys. Med. Imag., pp. 86680C1-14, 2013.

[22] M. A. C. Vieira, P. R. Bakic, A. D. A. Maidment, H. Schiabel, and N. A. D. Mascarenhas, "Filtering of poisson noise in digital mammography using local statistics and adaptive wiener filter," in Proc. 11th Int. Workshop Breast Imag., 2012, pp. 268-275.

[23] P. R. Bakic et al., "Comparison of 3D and 2D breast density estimation from synthetic ultrasound tomography images and digital mammograms of anthropomorphic software breast phantoms," Proc. SPIE 7961, Med. Imag., Phys. Med. Imag., p. 79610Z, 2011. 
[24] H. Yang, L. A. Christopher, N. Duric, E. West, and P. R. Bakic, "Performance analysis of EM-MPM and K-means clustering in 3D ultrasound image segmentation," in Proc. IEEE Int. Conf. Electro/Informa. Technol., 2012, pp. 1-4.

[25] A. B. Lau et al., "An anthropomorphic software breast phantom for tomosynthesis simulation: Power spectrum analysis of phantom reconstructions," Med. Phys., vol. 37, p. 3473, 2010.

[26] P. R. Bakic et al., "An anthropomorphic software breast phantom for tomosynthesis simulation: Power spectrum analysis of phantom projections," in Proc. 10th Int. Workshop Digital Mammogr., 2010, pp. 452-458.

[27] D. Kontos, C. Zhang, N. V. Ruiter, P. R. Bakic, and A. D. A. Maidment, "Evaluating the effect of tomosynthesis acquisition parameters on image texture: A study based on an anthropomorphic breast tissues software model," in Proc. 10th Int. Workshop Digital Mammogr., 2008, pp. 491-498.

[28] P. R. Bakic et al., "Testing realism of software breast phantoms: Texture analysis of synthetic mammograms," in Proc. SPIE 8668, Med. Imag., Phys. Med. Imag., 2013, pp. 8668241-12.

[29] S. Young et al., "Estimating breast tomosynthesis performance in detection tasks with variable-background phantoms," in Proc. SPIE 7250 Med. Imag., Phys. Med. Imag., 2009, p. 72580O.

[30] A. B. Lau, M. Das, and H. C. Gifford, "Towards visual-search model observers for mass detection in breast tomosynthesis," in Proc. SPIE 8668, Med. Imag., Phys. Med. Imag., 2013, p. 86680X.

[31] S. Young, P. R. Bakic, K. J. Myers, R. J. Jennings, and S. Park, "A virtual trial framework for quantifying the detectability of masses in breast tomosynthesis projection data," Med. Phys., vol. 40, no. 5, p. 051914, 2013.

[32] A.-K. Carton, H. Derand, C. Ullberg, P. R. Bakic, and A. D. A. Maidment, "Development of a 3D physical anthropomorphic breast phantom," presented at the Tomosynthesis Imag. Symp. 2009: Frontiers in Res. Clin. Appl., Durham, NC, 2009.

[33] A. K. Carton, P. R. Bakic, C. Ullberg, and A. D. A. Maidment, "Development of a 3D high-resolution physical anthropomorphic breast phantom," in Proc. SPIE 7622, Med. Imag., Phys. Med. Imag., 2010, p. 762206.

[34] A.-K. Carton, P. R. Bakic, C. Ullberg, H. Derand, and A. D. A. Maidment, "Development of a physical 3D anthropomorphic breast phantom," Med. Phys., vol. 38, no. 2, pp. 891-896, 2011.

[35] C. C. Brunner et al., "Evaluation of various mammography phantoms for image quality assessment in digital breast tomosynthesis," in Proc. 10th Int. Workshop Digital Mammogr., 2010, pp. 284-291.

[36] R. Karunamuni et al., "Exploring the relationship between SDNR and detectability in dual-energy breast x-ray imaging," in Proc. SPIE 8668, Med. Imag., Phys. Med. Imag., 2013, pp. 8668631-9.

[37] F. Chen et al., "Partial volume simulation in software breast phantoms," in Proc. SPIE 8313, Med. Imag., Phys. Med. Imag., 2012, pp. 83134U1-9.
[38] F. Chen et al., "Simulation of three material partial volume averaging in a software breast phantom," in Proc. 11th Int. Workshop Breast Imag., 2012, pp. 149-156.

[39] P. R. Bakic, C. Zhang, and A. D. A. Maidment, "Development and characterization of an anthropomorphic breast software phantom based upon region-growing algorithm," Med. Phys., vol. 38, no. 6, pp. 3165-3176, 2011.

[40] C. M. Li et al., "Computerized 3D breast phantom with enhanced highresolution detail," in Proc. SPIE 7258, Med. Imag., Phys. Med. Imag., 2009 , p. 72580 S.

[41] G. B. Folland, Advanced Calculus. Upper Saddle River, NJ: PrenticeHall, 2002.

[42] G. Thomas, Calculus and Analytic Geometry. New York: Dorling Kindersley, 2010.

[43] R. Y. Rubinstein and D. P. Kroese, Simulation and the Monte Carlo Method, 2nd ed. Hoboken, NJ: Wiley, 2007.

[44] P. J. Schneider and D. H. Eberly, Distance from point to a general quadratic curve or a general quadratic surface WA, Geometric Tools, LLC [Online]. Available: http://www.geometrictools.com/Documentation/DistancePointToQuadratic.pdf

[45] K. Pohlmann, Principles of Digital Audio. New York, NY: McGrawHill/TAB Electronics, 2010.

[46] A. Ringberg, E. Bageman, C. Rose, C. Ingvar, and H. Jernstrom, "Of cup and bra size: Reply to a prospective study of breast size and premenopausal breast cancer incidence," Int. J. Cancer, vol. 119, no. 9, pp. 2242-2243, 2006.

[47] T. L. Pope, M. E. Read, T. Medsker, A. I. Buschi, and A. N. Brenbridge, "Breast skin thickness: Normal range and cause of thickening shown on film-screen mammography," J. Can. Assoc. Radiol., vol. 35, no. 4, pp. 365-368, 1984.

[48] H. Ulger, N. Erdogan, S. Kumanlioglu, and E. Unue, "Effect of age, breast size, menopausal and hormonal status on mammographic skin thickness," Skin Res. Technol., vol. 9, no. 3, pp. 284-289, 2003.

[49] F. Contijoch, J. M. Lynch, D. D. Pokrajac, A. D. A. Maidment, and P. R. Bakic, "Shape analysis of dimulated breast anatomical structures," in Proc. SPIE 8313 Med. Imag., Phys. Med. Imag., 2012, p. 83134J.

[50] N. V. Ruiter et al., "Model-based registration of x-ray mammograms and MR images of the female breast," IEEE Trans Nucl. Sci., vol. 53, no. 1, pp. 204-211, Feb. 2006.

[51] J. H. Hubbel and S. M. Seltzer, Tables of X-ray mass attenuation coefficients and mass energy-absorption coefficients ver. 1.4. Gaithersburg, MD, Nat. Inst. Standards Technol., Jul. 2004 [Online]. Available: http://www.nist.gov/pml/data/xraycoef/index.cfm

[52] J. H. Chui, D. D. Pokrajac, A. D. A. Maidment, and P. R. Bakic, "Roadmap for efficient parallelization of breast anatomy simulation," in Proc. SPIE 8313 Med. Imag., Phys. Med. Imag., 2012, p. 83134T.

[53] J. H. Chui, D. D. Pokrajac, A. D. A. Maidment, and P. R. Bakic, "Toward breast anatomy simulation using GPU," in Proc. 11th Int. Workshop Breast Imag., 2012, pp. 506-513. 